\title{
Monte Carlo program KORALW 1.02 for $W$-pair production at LEP2/NLC energies with Yennie-Frautschi-Suura exponentiation ${ }^{\dagger}$
}

\author{
M. Skrzypek ${ }^{\star}$ \\ Institute of Nuclear Physics, Kraków, ul. Kawiory 26a, Poland \\ S. Jadach ${ }^{\star}$ \\ Institute of Nuclear Physics, Kraków, ul. Kawiory 26a, Poland, \\ and \\ CERN, Theory Division, Geneva 23, Switzerland \\ W. Płaczek ${ }^{\ddagger \star}$ \\ Department of Physics and Astronomy, \\ The University of Tennessee, Knoxville, Tennessee 37996-1200 \\ Z. W a̧s $^{\star}$ \\ CERN, Theory Division, Geneva 23, Switzerland, \\ and \\ Institute of Nuclear Physics, Kraków, ul. Kawiory 26a, Poland
}

\begin{abstract}
The Monte Carlo program KORALW version 1.02 for $W$-pair production is presented. Its main features are: multiple initial state photonic radiation with finite transverse photon momenta generated according to the Yennie-Frautschi-Suura Monte Carlo technique and massive kinematics for all final particles. Matrix element features second-order leading-logarithmic initial-state QED corrections.

The dedicated semi-analytical program KORWAN, with structure-function based leading-logarithmic initial-state photonic radiation up to third order, is also included in the distribution package.

To be submitted to Computer Physics Communications

$\dagger$ Work supported in part by Polish Government grant KBN 2P30225206, European Commission contract ERBCIPDCT940016 and IN2P3 French-Polish Collaboration through LAPP Annecy.

$\ddagger$ On leave of absence from Institute of Computer Science, Jagellonian University, Kraków, ul. Reymonta 4, Poland

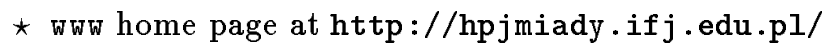

CERN-TH/95-205

July 1995 


\section{PROGRAM SUMMARY}

Title of the program: KORALW, version 1.02 .

Computer: HP-9000/735,

Operating system: UNIX,

Programming language used: FORTRAN 77

High speed storage required: $<1 \mathrm{MB}$

No. of bits in a word: 32

Peripherals used: Line printer

No. of cards in combined program and test deck: about 8988 plus $2272+5958$ of physic generators libraries.

Keywords: Radiative corrections, heavy boson $W$, Monte Carlo simulation, quantum electrodynamics, spin polarization, electro-weak theory, LEP2.

Nature of the physical problem: The $W$ pair production and decay will be used as an important data point for precise tests of the standard electroweak theory at LEP2 and higher energies. The effects due to QED bremsstrahlung and apparatus efficiency have to be subtracted from the data. The program includes also effects of the secondary decays, i.e. hadronization of quarks and $\tau$ lepton decays.

Method of solution: The Monte Carlo simulation of the combined $W$ production and decay process including subsequent decays of $\tau$ lepton and/or hadronization of quarks is used to calculate the effects of spin and the effects of radiative corrections, including hard bremsstrahlung, simultaneously. Any experimental cut and apparatus efficiency may be introduced easily by rejecting some of the generated events.

Restrictions on the complexity of the problem: See introduction

Typical running time: Efficiency is about $30 \mathrm{CPU}$ sec. of HP-9000/735/99 per 1000 unweighted events, for the parameter setting as in the demonstration output. 


\section{Contents}

1 Introduction $\quad 3$

2 The Monte Carlo Algorithm 5

2.1 Notation ..................... 5

2.2 Master Formula . . . . . . . . . . . . . . . . . . . 6

2.3 Crude Photonic Distribution . . . . . . . . . . . . . 7

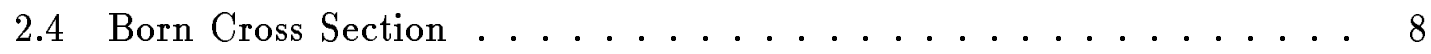

2.5 Crude Born ......................... 9

2.6 Normalization of the Crude Born ............... 11

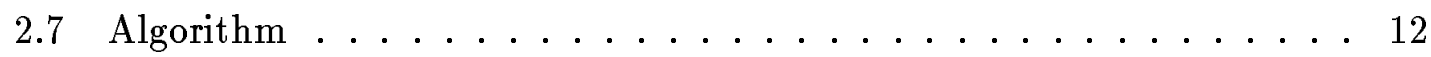

$2.8 \mathcal{R}$-Projection and Final-State Masses . . . . . . . . . . . 13

$2.9 \bar{\beta}$-Functions ...................... 15

3 Semi-analytical Calculation $\quad 16$

4 Structure of the Program $\quad 17$

4.1 korww . . . . . . . . . . . . . . . . . . . . . . 18

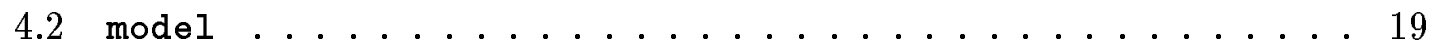

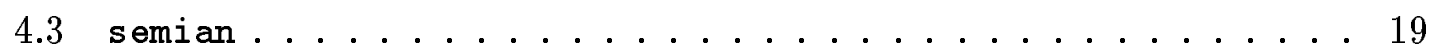

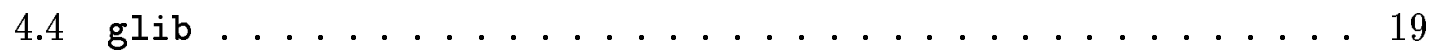

4.5 interfaces ..................... 20

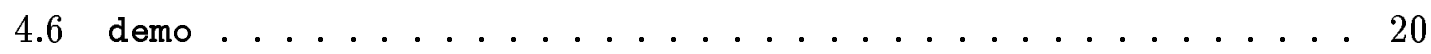

5 How to Use the Program? 21

5.1 Input/Output Parameters . . . . . . . . . . . . . . 21

5.2 Simple User Program . . . . . . . . . . . . . . . 21

6 Acknowledgements $\quad 28$

A Matrix Element $\quad 29$

B Listing of the Simple Program 33

C Output of the Simple Program $\quad 35$ 


\section{Introduction}

In the next years the LEP energy will be increased to pass the production threshold of the pair of $W$ bosons and we shall enter a new exciting era in the experimental tests of the electroweak Standard Model (SM). As is well known, the very successful Standard Model of electrowek interactions is deeply incomplete and we expect to find that it fails at certain moment, in confrontation with the experimental data. This would give us a hint to find its extension or replacement. The new experimental investigation of the $W$-pair production process at LEP is our next chance for this objective. In the new experiments at energies up to $200 \mathrm{GeV}$ in the centre of mass, we may find new particles or deviations in fine measurements of the total or differential cross section. In the second task of the maximally precise measurements of the total/differential cross section of the $W$-pair production and decay process we expect to reach the $1 \%$ experimental precision level. The corresponding theoretical predictions of the SM have to be given with at least $0.5 \%$ precision possibly at the $0.3 \%$ level. Furthermore, the $W^{-} W^{+}$production and decay process involves a complicated production mechanism with delicate gauge cancellations and multichannel $W$ decays into light leptons, heavy tau lepton and/or quark jets. The experimental measurements of the $W^{-} W^{+}$process will be complicated because of at least four particle/jet final states in the presence of experimental cuts. Also, due to limited statistics, it will be of critical importance to exploit maximally the detector efficiency/resolution. This inevitably means that high quality Monte Carlo event generator(s) for the $W$-pair production and decay process will be extremely helpful in the data analysis. For example, in the measurement of the $W$ mass, which will be one of the primary measurements at LEP, this $W$ mass will be measured either close to the threshold from the total cross section or further from the threshold, at the peak cross section, using final jet/lepton mass and/or angular distributions. For this and other measurements, elimination of all the effects due to the Initial State Radiation of photons (ISR) and proper evaluation of the apparatus inefficiencies will require extensive use of Monte Carlo (MC) event generators.

In this paper we present the first version of the Monte Carlo event generator KORALW, which is aimed to help the experimental data analysis for the $W$-pair production and decay process. The present version features:

- Matrix element for $W$-pair production and $W$-pair decay into four fermions (quarks and/or leptons) with proper $W$ spin treatment and finite $W$ width,

- Initial-state multi-photon emission with full photon phase space (i.e. with finite transverse photon momenta),

- Simmulation of the decay of polarized heavy $\tau$ lepton (decay product of $W$ ) in all possible channels, taking into account spin polarization and QED bremsstrahlung, 
- Emission by leptons, the decay products of $W$, of photons (up to double bremsstrahlung),

- Arrangement of quarks (decay products of $W$ ) into strings and fragmentation into hadrons according to the LUND model using JETSET,

- Massive kinematics with exact four momentum conservation for the entire $W^{-} W^{+}$production and decay process.

It is obvious from the above list that the present version of the program is already well advanced and may be useful for a realistic Monte Carlo simulation of the $W$ pair production and decay process. The most important limitations of the present program are:

- Lack of so-called "background process" Feynman diagrams; in particular Zpair production,

- Lack of optional additional "new physics" matrix elements,

- Simplified matrix element for QED photon emission,

- Lack of Coulomb correction close to threshold,

- Lack of electroweak non-QED corrections ${ }^{1}$,

- Simplified "colour arrangement" for four quark jets.

The above and other shortcomings of the program will be addressed in the next versions of the program.

The outline of the paper is the following: In section 2 we describe the matrix element for $W$-pair production and decay process and we lay down the MC algorithm of our MC program KORALW, which leads to efficient generation in spite of strong peaks in the differential distributions. In section 3 we briefly describe the simple semi-analytical calculation which is included in the package and was instrumental in debugging normalization of the total cross section of our KORALW at the technical (relative) precision level of $2 \times 10^{-4}$, see ref. [1]. In section 4 we describe the structure of the program, important subroutines, etc. In section 5 we instruct the reader on how to use the program, in particular we present and explain the simple "demo" program. The corresponding source codes and outputs are shown. After the paper, there are three appendices: (A) a description of the Born level matrix element used in the generation, (B) a listing of the demonstration program, (C) printouts from this demonstration program.

\footnotetext{
${ }^{1}$ Most probably these corrections are small in comparison with the experimental precision and it is not necessary to include them in the Monte Carlo program - it is enough if they are in the auxiliary semi-analytical program.
} 


\section{The Monte Carlo Algorithm}

In this section we present the Monte Carlo algorithm implemented in the KORALW program. The philosophy adopted by us in the construction of the algorithm can be summarized in the following steps:

- Write down the general, exact master formula for the cross section in terms of the standard Lorentz-invariant phase-space integration element times the appropriate matrix element. It can be arbitrarily complicated and must be, from the physics point of view, as complete as possible.

- Find a series of approximations of the master formula, until a fairly simple form is reached. The main constraint in this simplification is that we keep all the dominant singularities/peaks intact.

- Integrate analytically the crude distribution step by step until the so-called "crude" total cross section is reached.

- Going "backward" from the crude total cross section, find generation methods for all the necessary variables and reconstruct the Monte Carlo event in terms of four-momenta.

- Calculate the total cross section as the product of the average of the appropriate weights and the crude total cross section.

In the following subsections we discuss in detail the procedure outlined above for the KORALW Monte Carlo generator. We start with the complete, exact master formula for the total cross section. We explain it in detail and then introduce step by step all the simplifications that lead to the crude distribution, used by the generator. Finally we discuss the actual event generation algorithm. All the corresponding weights are also listed.

\subsection{Notation}

We adopt the following four-momenta notation convention: $p_{1}, p_{2}$ are the incoming $e^{-}, e^{+}$momenta with masses $m_{e} ; q_{1}, q_{2}$ are momenta of the decay products of the $W^{-}$ resonance, with $q_{1}$ being the fermion momentum and $q_{2}$ the antifermion momentum of the decay pair. Their masses are denoted by $m_{1}$ and $m_{2} ; q_{3}, q_{4}, m_{3}, m_{4}$ are the analogous momenta and masses of the $W^{+}$decay; $k_{i}$ are the momenta of the radiative photons. The components of a four-vector are denoted by $p=\left(p_{0}, \mathbf{p}\right)$. The invariants

$s$ and $s^{\prime}$ are defined in the usual way: $s=\left(p_{1}+p_{2}\right)^{2}$ and $s^{\prime}=\left(\sum_{i=1}^{4} q_{i}\right)^{2}$. 


\subsection{Master Formula}

Apart from the Born matrix element the photonic part of the master formula of KORALW is identical to that of YFS2 (see ref. [2]; YFS2 was also used in KORALZ [3]). Both are based on the principle of the Yennie-Frautschi-Suura (YFS) [4] resummation of the soft photons to all orders in perturbative QED. Let us note that in the presented version of KORALW only the Initial State Radiation (ISR) is considered whereas in the latest version of YFS3, ref. [5], also the Final State Radiation was included. We want to stress very clearly and strongly that the "truncation" of the YFS scheme to the leading-logarithmic version in the present work is not a genuine limitation of the YFS scheme but rather a temporary solution forced upon the authors by the fact that complete $\mathcal{O}\left(\alpha^{1}\right)$ QED matrix element for the $W$-pair production (with off-shell W's) is not yet available ${ }^{2}$. The master formula is the following

$$
\begin{aligned}
\sigma= & \sum_{n=0}^{\infty} \frac{1}{n !} \int \prod_{i=1}^{4} \frac{d^{3} q_{i}}{q_{i}^{0}}\left(\prod_{i=1}^{n} \frac{d^{3} k_{i}}{k_{i}^{0}} \tilde{S}\left(p_{1}, p_{2}, k_{i}\right)\right) \delta^{(4)}\left(p_{1}+p_{2}-\sum_{i=1}^{4} q_{i}-\sum_{i=1}^{n} k_{i}\right) \Theta_{\epsilon}^{c m} \\
& \exp \left(2 \alpha \Re B+\int \frac{d^{3} k}{k^{0}} \tilde{S}\left(p_{1}, p_{2}, k\right)\left(1-\theta_{\epsilon}^{c m}\right)\right)\left[\bar{\beta}_{0}^{(2)}\left(\mathcal{R} p_{1}, \mathcal{R} p_{2}, \mathcal{R} q_{1}, \mathcal{R} q_{2}, \mathcal{R} q_{3}, \mathcal{R} q_{4}\right)\right. \\
& +\sum_{\substack{i=1 \\
n}} \frac{\bar{\beta}_{1}^{(2)}\left(\mathcal{R} p_{1}, \mathcal{R} p_{2}, \mathcal{R} q_{1}, \mathcal{R} q_{2}, \mathcal{R} q_{3}, \mathcal{R} q_{4}, \mathcal{R} k_{i}\right)}{\tilde{S}\left(p_{1}, p_{2}, k_{i}\right)} \\
& \left.+\sum_{\substack{l, j=1 \\
l \neq j}}^{n} \frac{\bar{\beta}_{2}^{(2)}\left(\mathcal{R} p_{1}, \mathcal{R} p_{2}, \mathcal{R} q_{1}, \mathcal{R} q_{2}, \mathcal{R} q_{3}, \mathcal{R} q_{4}, \mathcal{R} k_{l}, \mathcal{R} k_{j}\right)}{\tilde{S}\left(p_{1}, p_{2}, k_{l}\right) \tilde{S}\left(p_{1}, p_{2}, k_{j}\right)}\right]
\end{aligned}
$$

where $\tilde{S}\left(p_{1}, p_{2}, k\right)=-\left(\alpha / 4 \pi^{2}\right)\left(\left(p_{1} / k p_{1}\right)-\left(p_{2} / k p_{2}\right)\right)^{2}$ is the real photon infrared (IR) factor and $\Theta_{\epsilon}^{c m}=\prod_{i=1}^{n} \theta\left(2 k_{i}^{0} / \sqrt{s}-\epsilon\right)$ cuts out the singular IR region, already included to all orders in the YFS form factor $F_{Y F S}$. The YFS form factor is equal to

$$
\begin{aligned}
F_{Y F S}\left(p_{1}, p_{2}, \epsilon\right) & =\exp \left[2 \alpha \Re B+\int \frac{d^{3} k}{k^{0}} \tilde{S}\left(p_{1}, p_{2}, k\right)\left(1-\theta\left(k^{0}-\frac{2 \epsilon}{\sqrt{s}}\right)\right)\right] \\
& =\exp \left[2 \frac{\alpha}{\pi}\left(\left(\ln \frac{s}{m_{e}^{2}}-1\right) \ln \epsilon+\frac{1}{2} \ln \frac{s}{m_{e}^{2}}-1+\frac{\pi^{2}}{3}\right)\right] .
\end{aligned}
$$

The $\bar{\beta}_{0}, \bar{\beta}_{1}$ and $\bar{\beta}_{2}$ functions will be discussed later on. For all the other details concerning eq. (1) we refer the reader to ref. [2].

\footnotetext{
${ }^{2}$ Examples of incomplete preliminary calculations can be found in refs. $[6,7]$.
} 


\subsection{Crude Photonic Distribution}

In the following few subsections we discuss simplifications of the master formula of eq. (1) leading to the crude distributions used as the basis of the actual MC generation. The series of simplifications of eq. (1) starts with the $\bar{\beta}$-terms. We drop completely the $\bar{\beta}_{1}$ and $\bar{\beta}_{2}$ contributions.

$$
\bar{\beta}_{1}^{(2)}, \bar{\beta}_{2}^{(2)} \rightarrow 0
$$

They are pure higher-order corrections, relatively small, and it is easy to restore them by reweighting events at the end of the MC generation.

The case of $\bar{\beta}_{0}$ is more complicated. To the lowest order, $\bar{\beta}_{0}$ is equal to the differential Born cross-section, and therefore has to be kept, at least to some approximation. ¿From now on we replace $\bar{\beta}_{0}$ by its lowest-order term:

$$
\begin{aligned}
\bar{\beta}_{0}^{(2)} \rightarrow \bar{\beta}_{0}^{(0)} & =\frac{1}{2 s^{\prime}} \mathcal{N}_{\pi}\left|\mathcal{M}_{B}\right|^{2}, \\
\mathcal{N}_{\pi} & =\frac{(2 \pi)^{4}}{\left(2(2 \pi)^{3}\right)^{r}},
\end{aligned}
$$

where $r$ denotes the number of Born level particles in the final state $(r=4$ in our case); see Sect. 2.4 for details on $\mathcal{M}_{B}$. With the help of the above approximations, see eqs. $(3,4)$, we get from eq. (1) the first crude distribution:

$$
\sigma_{c r u d e, 1}=F_{Y F S}\left(p_{1}, p_{2}, \epsilon\right) \sum_{n=0}^{\infty} \frac{1}{n !} \int\left(\prod_{i=1}^{n} \frac{d^{3} k_{i}}{k_{i}^{0}} \tilde{S}\left(k_{i}\right)\right) \Theta_{\epsilon}^{c m} \sigma_{B o r n}\left(s^{\prime}\right)
$$

with $\sigma_{B o r n}\left(s^{\prime}\right)$ denoting

$$
\begin{aligned}
\sigma_{\text {Born }}\left(s^{\prime}\right) & =\frac{1}{2 s^{\prime}} \int d \Phi_{4}\left|\mathcal{M}_{B}\right|^{2} \\
d \Phi_{4} & =(2 \pi)^{4} \delta^{(4)}\left(p_{1}+p_{2}-\sum_{i=1}^{n} k_{i}-\sum_{i=1}^{4} q_{i}\right) \prod_{i=1}^{4} \frac{d^{3} q_{i}}{2 q_{i}^{0}(2 \pi)^{3}},
\end{aligned}
$$

All these simplifications in $\bar{\beta}$ functions are reintroduced by the appropriate $w_{\beta}$ weight, defined as

$$
\begin{aligned}
w_{\beta}=\frac{2 s^{\prime}}{\mathcal{N}_{\pi}\left|\mathcal{M}_{B}\right|^{2}}[ & \bar{\beta}_{0}^{(2)}\left(\mathcal{R} p_{1}, \mathcal{R} p_{2}, \mathcal{R} q_{1}, \mathcal{R} q_{2}, \mathcal{R} q_{3}, \mathcal{R} q_{4}\right) \\
& +\sum_{i=1}^{n} \frac{\bar{\beta}_{1}^{(2)}\left(\mathcal{R} p_{1}, \mathcal{R} p_{2}, \mathcal{R} q_{1}, \mathcal{R} q_{2}, \mathcal{R} q_{3}, \mathcal{R} q_{4}, \mathcal{R} k_{i}\right)}{\tilde{S}\left(p_{1}, p_{2}, k_{i}\right)} \\
& \left.+\sum_{\substack{l, j=1 \\
l \neq j}}^{n} \frac{\bar{\beta}_{2}^{(2)}\left(\mathcal{R} p_{1}, \mathcal{R} p_{2}, \mathcal{R} q_{1}, \mathcal{R} q_{2}, \mathcal{R} q_{3}, \mathcal{R} q_{4}, \mathcal{R} k_{l}, \mathcal{R} k_{j}\right)}{\tilde{S}\left(p_{1}, p_{2}, k_{l}\right) \tilde{S}\left(p_{1}, p_{2}, k_{j}\right)}\right] .
\end{aligned}
$$


Now we reduce the photonic part of eq. (5) to a single integral over $v$ variable. This procedure and algorithm for the generation of bremsstrahlung photon kinematics for a given $v$ is described in detail in ref. [2]. For that reason we skip here all the details of the evaluation and refer the interested reader directly to this work for more information. Here we confine ourselves to the final result of this simplification.

After this simplification our $\sigma_{c r u d e, 1}$ distribution is reduced to:

$$
\sigma_{\text {crude }, 2}=e^{\delta_{Y F S}} \int_{0}^{v_{\max }} d v \gamma v^{\gamma-1} \rho(v) \sigma_{\text {Born }}((1-v) s) .
$$

The $v$ variable is defined as $v=1-s^{\prime} / s$. The function $\rho(v)$ is defined as

$$
\begin{aligned}
\rho(v) & =\frac{1}{2}\left(1+\frac{1}{\sqrt{1-v}}\right)\left(\theta(\epsilon-v)+\theta(v-\epsilon) \frac{\gamma^{\prime}}{\gamma}\left(\frac{v}{\epsilon}\right)^{\gamma^{\prime}-\gamma}\right), \\
\gamma & =2 \frac{\alpha}{\pi}\left(\ln \frac{s}{m_{e}^{2}}-1\right), \\
\gamma^{\prime} & =2 \frac{\alpha}{\pi} \ln \frac{s}{m_{e}^{2}} \\
\delta_{Y F S} & =\frac{\alpha}{\pi}\left(\frac{1}{2} \ln \frac{s}{m_{e}^{2}}-1+\frac{\pi^{2}}{3}\right) .
\end{aligned}
$$

Details corresponding to the $\sigma_{c r u d e, 2} \rightarrow \sigma_{\text {crude, } 1}$ restoration, which include the generation of bremsstrahlung photon complete kinematics, are described in detail in ref. [2]. Let us note that the corresponding weight will be denoted as

$w_{Y F S} \cdot$

\subsection{Born Cross Section}

The matrix element for $W$-pair production at LEP2 of eq. (6) is a rather complicated object. For practical reasons it is usually written not in terms of the close analytical formula, but rather in terms of spin amplitudes calculated numerically, see e.g. refs. $[8,9]$. On the other hand the Born cross-section exhibits a strongly varying energy dependence ( $W W$ threshold) as well as a non-trivial angular behaviour ( $s$ and $t$ channels together); this, for the sake of constructing an efficient $\mathrm{MC}$ algorithm, has to be understood, i.e. some analytical (at least approximate) expressions are of interest. Let us discuss the distributions in eq. (6) in detail and find some approximate formula reflecting basic properties of the integrand in eq. (6).

We start by rewriting the expression for the Born cross section of eq. (6), showing explicitly the sum over all the open decay channels $(a, b)$. We assume implicit summation over final-state spin states and average over initial-state spin:

$$
\sigma_{\text {Born }}\left(s^{\prime}\right)=\frac{1}{2 s^{\prime}} \sum_{\substack{a, b \\ \text { decay } \\ \text { chan. }}} \int d \Phi_{4}\left|\mathcal{M}_{a b}\right|^{2} .
$$


Standard manipulations on the four-body phase-space lead to the expression

$$
\begin{aligned}
d \Phi_{4}= & \frac{1}{32} d s_{1} d s_{2} d \Omega d \Omega_{1} d \Omega_{2} \mathcal{J} \Theta_{S} \mathcal{N}_{\pi}, \\
\mathcal{J}= & \sqrt{\lambda\left(1, \frac{s_{1}}{s^{\prime}}, \frac{s_{2}}{s^{\prime}}\right)} \sqrt{\lambda\left(1, \frac{m_{1}^{2}}{s_{1}}, \frac{m_{2}^{2}}{s_{1}}\right)} \sqrt{\lambda\left(1, \frac{m_{3}^{2}}{s_{2}}, \frac{m_{4}^{2}}{s_{2}}\right)}, \\
\lambda(x, y, z)= & x^{2}+y^{2}+z^{2}-2 x y-2 x z-2 y z \\
\Theta_{S}= & \theta\left(s^{\prime}-s_{1}\right) \theta\left(\sqrt{s^{\prime}}-\sqrt{s_{1}}-\sqrt{s_{2}}\right) \\
& \theta\left(\sqrt{s_{1}}-m_{1}-m_{2}\right) \theta\left(\sqrt{s_{2}}-m_{3}-m_{4}\right),
\end{aligned}
$$

where $\Omega$ is a solid angle of the $W^{-}$in the CMS frame ${ }^{3}$ with the $+z$-axis pointing in the direction of the incoming $e^{-}$beam, while $\Omega_{1(2)}$ is the solid angle of the $W^{-(+)}$ decay products in the $W^{-(+)}$rest frame. The $s_{1(2)}$ denote invariant mass squared of the virtual $W^{-(+)}$. The detailed description of the matrix element $\left|\mathcal{M}_{a b}\right|^{2}$ is given in Appendix A.

\subsection{Crude Born}

In the next step we have to simplify $d \Phi_{4}$ and the matrix element $\left|\mathcal{M}_{a b}\right|^{2}$ into a form suitable for the Monte Carlo generation: $\left|\mathcal{M}_{a b}\right|^{2} \rightarrow\left|\mathcal{M}_{a b}\right|_{\text {crude }}^{2}$. As a result of some numerical trials we have chosen the following representation for the "crude matrix element" 4

$$
\begin{aligned}
\left|\mathcal{M}_{a b}\right|_{c r u d e}^{2} & =N_{a b} \frac{1}{\mathcal{N}_{\pi}} \sigma_{0}\left(s^{\prime}\right) W\left(s_{1}\right) W\left(s_{2}\right) T(\cos \theta) C\left(\cos \theta_{1}\right) C\left(\cos \theta_{2}\right), \\
N_{a b} & =\frac{B R_{a}}{B R_{(e \nu)}} \frac{B R_{b}}{B R_{(e \nu)}}, \sum_{i} B R_{i}=1 \\
W\left(s_{i}\right) & =\frac{1+\left(s_{i} / M_{W}^{2}-1\right) \theta\left(s_{i}-M_{W}^{2}\right)}{B W^{0}\left(s_{i}\right)} \\
B W^{0}\left(s_{i}\right) & =\left(s_{i}-M_{W}^{2}\right)^{2}+\left(\Gamma_{W} M_{W}\right)^{2} \\
T(\cos \theta) & =\left[\frac{1}{2}\left(\left(s^{\prime}-s_{1}-s_{2}\right)-\sqrt{\lambda\left(s^{\prime}, s_{1}, s_{2}\right)} \cos \theta\right)\right]^{-1} \\
C\left(\cos \theta_{i}\right) & =\frac{1+\Delta\left(s^{\prime}\right)+\cos \theta_{i}}{1+\Delta\left(s^{\prime}\right)} .
\end{aligned}
$$

\footnotetext{
${ }^{3}$ In the actual FORTRAN code of KORALW we assume $\Omega$ to be in the $\mathrm{CMS}^{\prime}$ frame of the effective beams. This choice reflects part of the freedom we have in reducing the multiphotonic phase space into the Born phase space; see Sect. 2.8 for details.

${ }^{4}$ The purpose of this "crude matrix element" is purely technical. Strictly speaking it is to generate flat $d \Phi_{4}$ phase space weighted events with the weight including the $1 /\left|M_{a b}\right|_{\text {crude }}^{2}$ factor necessary for compensating matrix element peaks.
} 
The factor $N_{a b}$ is the relative normalization of the different decay channels with respect to the $(e \bar{\nu}, \bar{e} \nu)$ channel. The $B R_{a}$ is the branching ratio for the channel $a$. Except for the $N_{a b}$, the remaining part of eq. (17) is the same for all the decay channels. The angles $\theta, \phi$ and $\theta_{i}, \phi_{i}$ correspond to the solid angles $\Omega$ and $\Omega_{i}$ of eq. (13). The $\sigma_{0}\left(s^{\prime}\right)$ and $\Delta\left(s^{\prime}\right)$ are some complicated functions of $s^{\prime}$ optimized to approximate the true $s^{\prime}$ behaviour of the matrix element ${ }^{5}$. To the above simplifications of the matrix element corresponds the weight $w_{B}$ defined as

$$
w_{B} \cdot w_{B W}=\frac{\left|\mathcal{M}_{a b}\right|^{2}}{\left|\mathcal{M}_{a b}\right|_{c r u d e}^{2}}
$$

where the $w_{B W}$ weight explicitly accounts for the fact that the $W$ propagator BreitWigner functions $B W\left(s_{i}\right)$ defined in Appendix A are replaced in the crude Born distribution by the "non-running" $B W^{0}\left(s_{i}\right)$

$$
B W\left(s_{i}\right) \rightarrow B W^{0}\left(s_{i}\right)
$$

The appropriate $w_{B W}$ weight factor is defined as

$$
w_{B W}=\frac{B W^{0}\left(s_{1}\right)}{B W\left(s_{1}\right)} \frac{B W^{0}\left(s_{2}\right)}{B W\left(s_{2}\right)} .
$$

The next approximations are the phase-space limits on the $s_{1}, s_{2}$ variables, denoted collectively as $\Theta_{S}$ in eq. (13). We could in principle generate $s_{1}, s_{2}$ in the exact $\Theta_{S}$ domain. For the sake of simplicity of the algorithm, however, we replace $\Theta_{S}$ by a broader and easier to handle integration domain defined by the function $\Theta_{S}^{c r u d e}$

$$
\Theta_{S}^{c r u d e}=\theta\left(s-s_{1}\right) \theta\left(s_{\text {max }}-s_{2}\right), s_{\max }=s \theta\left(\frac{s^{\prime}}{4}-s_{1}\right)+\frac{s^{\prime}}{4}\left(1-\theta\left(\frac{s^{\prime}}{4}-s_{1}\right)\right) .
$$

The corresponding weight, denoted by $w_{S}$, is defined as

$$
w_{S}=\Theta_{S}
$$

Finally, to make the crude $\sigma_{\text {Born }}$ distribution completely factorizable into independent distributions over $s_{1}, s_{2}$ and angles, the $\mathcal{J}$ function of eq. (14) is approximated by 1 and the corresponding weight we denote by $w_{\mathcal{J}}$, defined as

$$
w_{\mathcal{J}}=\mathcal{J}\left(s^{\prime}, s_{1}, s_{2}\right)
$$

\footnotetext{
${ }^{5}$ Let us remember that the actual form of the functions $\Delta\left(s^{\prime}\right)$ and $\sigma_{0}\left(s^{\prime}\right)$ is irrelevant for the final distributions and total cross sections, as it cancels out with the introduction of the weight $w_{B} \cdot w_{B W}$. It may reflect only upon the efficiency of the MC generation.
} 
Putting together all the above simplifications we end up with the following crude cross section

$$
\begin{aligned}
\sigma_{\text {Born }}^{\text {crude }}\left(s^{\prime}\right)= & \left(\sum_{a, b} N_{a b}\right) \frac{1}{32} \frac{1}{2 s^{\prime}} \sigma_{0}\left(s^{\prime}\right) \int_{0}^{s} d s_{1} \int_{0}^{s_{\max }} d s_{2} W\left(s_{1}\right) W\left(s_{2}\right) \\
& \int_{0}^{2 \pi} d \phi \int_{-1}^{1} d \cos \theta T(\cos \theta) \\
& \int_{0}^{2 \pi} d \phi_{1} \int_{-1}^{1} d \cos \theta_{1} C\left(\cos \theta_{1}\right) \int_{0}^{2 \pi} d \phi_{2} \int_{-1}^{1} d \cos \theta_{2} C\left(\cos \theta_{2}\right),
\end{aligned}
$$

which, as we remember, is the simplified form of $\sigma_{\text {Born }}$ of eq. (12).

\subsection{Normalization of the Crude Born}

The crude Born matrix element of eq. (29) was chosen in such a way that it can easily be integrated analytically. This is crucial for the generation of the phase-space points. The overall normalization is determined by the crude cross section which is known analytically. Specifically, one can immediately integrate out all the angular degrees of freedom in eq. (29) with the overall result $(4 \pi)^{3} / 2$ :

$$
\sigma_{\text {Born }}^{\text {crude }}\left(s^{\prime}\right)=\frac{(4 \pi)^{3}}{2}\left(\sum_{a, b} N_{a b}\right) \frac{1}{32} \frac{1}{2 s^{\prime}} \sigma_{0}\left(s^{\prime}\right) \int_{0}^{s} d s_{1} \int_{0}^{s_{\max }} d s_{2} W\left(s_{1}\right) W\left(s_{2}\right) .
$$

Also the integration over $s_{1}$ and $s_{2}$ can be carried out analytically, yielding the result $S\left(s^{\prime}\right)$, while resummation over the decay channels $(a, b)$ gives $\sum_{a, b} N_{a b}=1 /\left(B R_{(e \nu)}\right)^{2}$

$$
\begin{aligned}
\sigma_{\text {Born }}^{\text {crude }}\left(s^{\prime}\right)= & \frac{\pi^{3}}{\left(B R_{(e \nu)}\right)^{2}} \frac{1}{2 s^{\prime}} \sigma_{0}\left(s^{\prime}\right) S\left(s^{\prime}\right) \\
S\left(s^{\prime}\right)= & A_{1}\left(s^{\prime}\right)^{2}+2 A_{1}\left(s^{\prime}\right) A_{2}\left(s^{\prime}\right) \\
A_{1}\left(s^{\prime}\right)= & w\left(\frac{s^{\prime}}{4}\right)-w(0)+\theta\left(\frac{s^{\prime}}{4}-M_{W}^{2}\right)\left[u\left(\frac{s^{\prime}}{4}\right)-u\left(M_{W}^{2}\right)\right] \\
A_{2}\left(s^{\prime}\right)= & w(s)-w\left(\frac{s^{\prime}}{4}\right)+\theta\left(s-M_{W}^{2}\right)[u(s) \\
& \left.-\theta\left(\frac{s^{\prime}}{4}-M_{W}^{2}\right) u\left(\frac{s^{\prime}}{4}\right)-\left(1-\theta\left(\frac{s^{\prime}}{4}-M_{W}^{2}\right)\right) u\left(M_{W}^{2}\right)\right] \\
w(s)= & \frac{1}{\Gamma_{W} M_{W}} \arctan \left(\frac{s-M_{W}^{2}}{\Gamma_{W} M_{W}}\right) \\
u(s)= & \frac{1}{2 M_{W}^{2}} \log \left(\left(s-M_{W}^{2}\right)^{2}+\left(\Gamma_{W} M_{W}\right)^{2}\right) .
\end{aligned}
$$

This completes the evaluation of the Born contribution to the master formula as well as different level "crude" distributions. 


\subsection{Algorithm}

At this point we are ready to insert the integrated crude Born of eq. (31) into the crude photonic formula of eq. (8) and write down the final one-dimensional crude distribution:

$$
\sigma_{\text {crude }}=e^{\delta_{Y F S}} \frac{\pi^{3}}{2} \frac{1}{\left(B R_{(e \nu)}\right)^{2}} \int_{0}^{v_{\max }} d v \gamma v^{\gamma-1} \rho(v) \frac{\sigma_{0}((1-v) s)}{(1-v) s} S((1-v) s) .
$$

Starting from the above formula, the actual generation is done in the following steps:

1. The variable $v$, the central variable of the Monte Carlo algorithm, is generated according to the one-dimensional $v$-distribution of eq. (32) with the help of the general-purpose Monte Carlo sampler (subroutine vesk1w). The auxiliary weight set by vesk1 1 in the course of constructing distribution (32) is denoted by

$w_{V E S}$.

2. All variables necessary for the construction of a complete kinematic configuration of real bremsstrahlung photons are generated identically as in the algorithm of YFS2 program, see ref. [2]. The appropriate additional factor $w_{Y F S}$ is calculated. We refer the interested reader to ref. [2] for more details.

3. In the next step we choose the decay channels $(a, b)$. This is done according to the branching ratios hidden in the function $N_{a b}$, defined in eq. (17); see also eq. (30).

4. Now, having fixed the decay channels and the variable $v$, we generate variables which will lead, after introduction of an appropriate weight, to a parametrization of the four-body phase space (see eq.(13)). We start with the distribution (30). The $s_{1}, s_{2}$ variables are generated according to the two-dimensional function $W\left(s_{1}\right) W\left(s_{2}\right) \Theta_{S}^{\text {crude }}$.

5. Next we generate the angular distributions of eq. (29). First the $\theta$ and $\phi$ variables are generated according to the $T(\cos \theta)$ function defined in eq. (21).

6. Finally, the angles $\theta_{i}$ and $\phi_{i}$ are generated according to the $C\left(\cos \theta_{i}\right)$ distributions of eq. (22). This completes the generation of the kinematic variables given in eq. (29), and in fact of all the variables necessary to reconstruct the whole event.

The total Monte Carlo weight $w$ is a product of all the intermediate weights listed earlier. We have separated them in two groups: technical, $w_{\text {Crude }}$, related to the 
construction of the crude Monte Carlo algorithm, eqs. $(33,11,27,25)$, and physical, $w_{\text {Model }}$, related to the matrix element used, eqs. $(23,28,7)$

$$
w=w_{C r u d e} w_{M o d e l}, \quad w_{C r u d e}=w_{V E S} w_{Y F S} w_{S} w_{B W}, \quad w_{M o d e l}=w_{B o r n} w_{\beta}
$$

Furthermore, for practical reasons we merged the $w_{B}$ and $w_{\mathcal{J}}$ weights into one weight denoted $w_{\text {Born }}$

$$
w_{\text {Born }}=w_{B} w_{\mathcal{J}}
$$

Finally, let us note that a shift of the overall normalization constants, such as factors $\pi$ or coupling constants, was executed, in the actual FORTRAN code, between $\left|\mathcal{M}_{a b}\right|^{2}, \sigma_{0}(s)$ used in the differential distribution and $\sigma_{0}(s)$ used in formula (32).

\section{$2.8 \mathcal{R}$-Projection and Final-State Masses}

We owe the reader some explanation about the meaning of the symbol $\mathcal{R}$ used frequently in connection with the $\bar{\beta}$-functions, see e.g. eq. (1).

The $\mathcal{R}$-operation is a projection operation from the multiphotonic phase space onto the four-body phase space with no photons. Some kind of operation of this type is a part inherent in the YFS scheme in which the $\bar{\beta}_{i}, i=1 \ldots n$ functions are defined for the phase space with $n$ photons. It simply reflects the fact that the $\bar{\beta}_{i}, i=1 \ldots n$, functions are the residua after subtraction of the singular $\tilde{S}$ factors, and strictly speaking they are defined at the singularity point $k_{j}=0, j>n$. In general such a procedure has to extrapolate a matrix element, as defined from Feynman rules, into phase space with additional real (even hard) photons. The $\mathcal{R}$-operation is a particular realization of such an extrapolation, which consists of some manipulations on four-momenta in order to remove additional photons (keeping four-momentum conservation). It is also sometimes called the "technique of effective beam". It was used in the YFS2/YFS3 programs and it has certain advantages. However, it is not the only possible solution (and not the best one); for example, in the BHLUMI program of ref. [10] the extrapolation was done without referring to the technique of the effective beams, but simply by extending the validity of the analytical expressions for the matrix element to higher-dimensional phase space.

Also note that in our present implementation $\mathcal{R}$-projections for $\bar{\beta}_{1}$ and $\bar{\beta}_{2}$ are reduced/related completely to the $\mathcal{R}$-projection for $\bar{\beta}_{0}$. In general it is not true and here it was possible only because the present approach is restricted to the leadinglogarithmic approximation. It must also be remembered that the $\tilde{S}$ factors are never affected by $\mathcal{R}$-projections or extrapolation.

How is the $\mathcal{R}$-operation implemented in KORALW? The final-state $\mathcal{R} q_{i}$ momenta are simply the $q_{i}$ momenta transformed from the CMS frame to the new frame CMS'. This new frame is defined as the rest frame of the $W^{-} W^{+}$pair. The orientation of

the CMS' frame is defined with the help of "effective beams" $p_{i}^{e f f}$. The $+z$ direction 
of $\mathrm{CMS}^{\prime}$ is oriented along the $\mathbf{p}_{1}^{\text {eff }}$ three-vector. The "effective beams" $p_{i}^{\text {eff }}$ are defined in the CMS frame as

$$
p_{1}^{e f f}=p_{1}-\sum_{\substack{i \\ k_{3} \geq 0}} k_{i}, p_{2}^{e f f}=p_{2}-\sum_{\substack{i \\ k_{3}<0}} k_{i} .
$$

To construct the $\mathrm{CMS}^{\prime}$ frame we transform the $p_{i}^{\text {eff }}$ to their rest frame. It is done by means of a boost along the vector $v=p_{1}^{e f f}+p_{2}^{e f f}$. The actual Lorentz transformation is the standard one: $x_{0}^{\prime}=\gamma\left(x_{0}-\boldsymbol{\beta} \mathbf{x}\right), \mathbf{x}^{\prime}=\mathbf{x}+\left((\gamma-1) / \boldsymbol{\beta}^{2}\right)(\boldsymbol{\beta} \mathbf{x}) \boldsymbol{\beta}-\gamma \boldsymbol{\beta} x_{0}$, $\boldsymbol{\beta}=\mathbf{v} /\left(\gamma m_{v}\right), \gamma=1 / \sqrt{1-\boldsymbol{\beta}^{2}}$, see ref. [11]. To complete the construction we rotate the $p_{i}^{e f f}$ vectors around the $y$ - and $x$-axes so that the $\mathbf{p}_{1}^{\text {eff }}$ would point into the $+z$ direction. We call this frame the CMS' frame and all other four-vectors are transformed from CMS to $\mathrm{CMS}^{\prime}$ in the same way. Finally, we redefine the effective beams in the $\mathrm{CMS}^{\prime}$ frame to be $p_{1}^{\text {eff }}=\left(\sqrt{s^{\prime}} / 2,0,0, \sqrt{s^{\prime}} / 2\right)$ and $p_{2}^{\text {eff }}=\left(\sqrt{s^{\prime}} / 2,0,0,-\sqrt{s^{\prime}} / 2\right)$. This set of $\mathrm{CMS}^{\prime}$ momenta is used to compute the matrix element.

The actual KORALW algorithm reverses in some sense the procedure described above. Namely, in the course of constructing the Monte Carlo event KORALW assumes that all the angles $\theta$ and $\phi$ are defined in the $\mathrm{CMS}^{\prime}$ frame, i.e. they refer directly to the $\mathcal{R}$-projected final states. Instead, the transformation back to CMS involves the $\mathcal{R}$-procedure.

The other issue we want to address here is the way we adopt the massive finalstate kinematics for the massless matrix element. We have to keep in mind that in principle one can use the complete, massive matrix element. In fact the KORALW program is prepared for that option. We regard it, however, as an unnecessary complication of the program, affecting the results well below the required accuracy of LEP2. Therefore, we stick to the relatively simple massless matrix element. To estimate the accuracy of this approximation we provide two different mass-reduction prescriptions on how to transform massive four-vectors into massless ones. The first, "brute-force" method makes final-state fermions massless by simple rescaling of their three-momenta. Rescaling by the factor $q_{i}^{0} /\left|\mathbf{q}_{\mathbf{i}}\right|$ is done in the CMS' frame, i.e. in the CMS frame of the effective beams. Of course this procedure violates the momentum conservation. The other, "sophisticated" method conserves fourmomentum and preserves all the angles. In practice it is implemented by repeating the construction done for the true, massive final states, starting from the same set of angles $\theta, \phi, \theta_{1}, \phi_{1}, \theta_{2}, \phi_{2}$ of eqs. $(13,29)$ but with substituted zero masses. Since these two mass-reduction procedures are so different, switching between them provides a good estimate of the size of the mass effect.

Two more comments are in order here. First, we want to stress that both these mass-reduction procedures are the internal parts of the matrix element calculation and, in other parts of the program, such as in the calculation of phase-space Jacobians, true massive kinematics is used. Therefore, not only kinematic configurations generated by our program will differ from the massless case, but so will the differential and total cross sections, even if the analytical form of the matrix elements is 
identical to the massless case.

\section{$2.9 \bar{\beta}$-Functions}

The $\mathcal{O}\left(\alpha^{r}\right)$ functions $\bar{\beta}_{i}^{(r)}$ are defined in the Yennie-Frautschi-Suura scheme [4] as residuals after the removal of infrared virtual and real singularities. They are therefore infrared-finite. In the YFS scheme they are obtained from the $\mathcal{O}\left(\alpha^{r}\right)$ "raw" differential distributions which originate from Feynman diagrams. This is the case in the $\mathcal{O}\left(\alpha^{1}\right)$ YFS-exponentiated matrix element of BHLUMI $[10,12,13]$ or YFS2 [2] and YFS3 [5]. In the $\mathcal{O}\left(\alpha^{2}\right)$ the "raw" distributions are often constructed as a leading-logarithmic (LL) ansatz $[2,13]$ instead of being taken directly from the Feynman rules ${ }^{6}$. Here, in our calculation we employ an LL ansatz not only in $\mathcal{O}\left(\alpha^{2}\right)$ but already in $\mathcal{O}\left(\alpha^{1}\right)$. Let us stress immediately that we treat our present LL ansatz as a temporary solution on the way to a better $\mathcal{O}\left(\alpha^{1}\right)$ matrix element. In this way we set already now a well-defined framework for future development of the program. The other advantage of our scheme is that events simulated in our present version of the MC program feature realistic multiple photons with finite transverse momenta. Two things have to be borne in mind: (a) in our matrix element the $\mathcal{O}\left(\alpha^{1}\right)$ nonlogarithmic contributions are already present in the YFS form factor ${ }^{7}$, see eq. (10); (b) the $\gamma$ parameter in eq. (9), contrary to other LL approaches, is not inserted by hand but results from the phase-space integration.

The actual expressions for $\bar{\beta}_{i}^{(r)}, r=1,2$ functions are defined:

$$
\begin{aligned}
& \bar{\beta}_{0}^{(1)}=\bar{\beta}_{0}^{(0)}(1+\gamma / 2), \quad \bar{\beta}_{0}^{(2)}=\bar{\beta}_{0}^{(0)}\left(1+\gamma / 2+\gamma^{2} / 8\right), \\
& \bar{\beta}_{1}^{(1)}\left(k_{i}\right)=\bar{\beta}_{0}^{(0)} \nu\left(x_{i}\right) \tilde{S}\left(p_{1}, p_{2}, k_{i}\right), \\
& \bar{\beta}_{1}^{(2)}\left(k_{i}\right)=\bar{\beta}_{0}^{(0)}\left(k_{i}\right)\left[\nu\left(x_{i}\right)+(\gamma / 4) \chi\left(x_{i}\right) \ln \left(1-x_{i}\right)\right] \tilde{S}\left(p_{1}, p_{2}, k_{i}\right), \\
& \bar{\beta}_{2}^{(2)}\left(k_{i}, k_{j}\right)=\bar{\beta}_{0}^{(0)}\left(k_{i}, k_{j}\right)\left\{\theta\left(-k_{i}^{3} k_{j}^{3}\right)\left[\chi\left(x_{i}\right) \chi\left(x_{j}\right)-\nu\left(x_{i}\right)-\nu\left(x_{j}\right)-1\right]\right. \\
& \left.\quad+\theta\left(k_{i}^{3} k_{j}^{3}\right)\left[\chi\left(x_{i}\right) \chi\left(x_{j}^{*}\right) / 2+\chi\left(x_{i}^{*}\right) \chi\left(x_{j}\right) / 2-\nu\left(x_{i}\right)-\nu\left(x_{j}\right)-1\right]\right\} \\
& \quad \times \tilde{S}\left(p_{1}, p_{2}, k_{i}\right) \tilde{S}\left(p_{1}, p_{2}, k_{j}\right),
\end{aligned}
$$

where $\bar{\beta}_{0}^{(0)}$ is defined in eq. (4), $x_{i}=k_{i}^{0} / E_{\text {beam }}$, and in the $\bar{\beta}_{2}$ definition we have to employ $x_{i}^{*}=x_{i} /\left(1-x_{j}\right), i \neq j$. The function $\theta\left(-k_{i}^{3} k_{j}^{3}\right)$ is non-zero for photons in the opposite hemispheres defined with respect to the initial beams, and similarly the function $\theta\left(k_{i}^{3} k_{j}^{3}\right)$ is non-zero for photons in the same hemisphere.

It is straightforward to check, by expanding the YFS form factor of eq. (10) and truncating all our differential distributions to $\mathcal{O}\left(\alpha^{r}\right) r=1,2$, that the dependence

\footnotetext{
${ }^{6}$ This solution is simple and economical in terms of the size (computation time) and of the resulting physical precision.

${ }^{7}$ For the sake of comparison with other programs, we provide a switch in the input data which sets to zero the $\mathcal{O}\left(\alpha^{1}\right)$ subleading terms in the YFS form factor.
} 
of the distributions on the longitudinal photon momenta follows exactly the LL QED behaviour in the corresponding order; see for example the LL QED structure functions of ref. [14].

Note that in the present LL version of the QED matrix element the reduction procedure $\mathcal{R}$ described in the previous subsection is hidden entirely in $\bar{\beta}_{0}^{(0)}$, that is in the Born differential cross section. The soft factors $\tilde{S}$ in eq. (37) are not concerned by the reduction procedure.

\section{Semi-analytical Calculation}

The semi-analytical calculation implemented in the KORWAN routine is based on the formulas for the Born cross section taken from ref. [15]. The following modifications had to be done in order to match the normalization of KORALW: introduction of the $Z$ width to the $Z$ propagator, change of the Breit-Wigner functions of the $W$ resonances to the form $B W\left(s_{i}\right)$ of eq. (46), and appropriate changes in the overall normalization scheme. The ISR corrections are implemented via the LL structure functions (SF) approach. The complete formula is given by the convolution of the two SF's with the Born cross section

$$
\sigma^{S A N}\left(v_{\min }, v_{\max }\right)=N_{S} \int_{1-v_{\max }}^{1-v_{\min }} d x F(x, s) \int_{0}^{x s} d s_{1} \int_{0}^{\left(\sqrt{x s}-\sqrt{s_{1}}\right)^{2}} d s_{2} \rho\left(s_{1}\right) \rho\left(s_{2}\right) \sigma_{0}^{S}\left(x s, s_{1}, s_{2}\right)
$$

with

$$
\begin{aligned}
\rho\left(s_{i}\right) & =\frac{1}{\pi} \frac{1}{12} \frac{\alpha_{W}}{\sin ^{2} \theta_{W}} \frac{s_{i}}{B W\left(s_{i}\right)}, \\
N_{S} & =\left(\frac{1}{B R_{(e \nu)}}\right)^{2}, \\
F(x, s) & =\int_{0}^{1} d x_{1} d x_{2} \delta\left(x-x_{1} x_{2}\right) D\left(x_{1}, s\right) D\left(x_{2}, s\right) .
\end{aligned}
$$

The Breit-Wigner $\rho\left(s_{i}\right)$ function of the $W$ resonances is modified to agree with the KORALW choice - $B W\left(s_{i}\right)$. The overall normalization factor $N_{S}$ is adjusted so that the $\sigma_{0}^{S}\left(x s, s_{1}, s_{2}\right)$ function, for the cross section summed over all channels, is identical to the function $\sigma\left(x s, s_{1}, s_{2}\right)$ of eq. (4) ref. [15]. The only modification added to $\sigma_{0}^{S}$ with respect to $\sigma$ is the introduction of the $Z$ width to the $Z$ propagators in $\sigma_{0}^{S}$. The $\alpha_{W}$ denotes the QED coupling constant at $W W$ threshold and $\sin \theta_{W}$ is the sine of the Weinberg angle.

The function $F(x, s)$ is the convolution of the two electron non-singlet structure functions $D\left(x_{i}, s\right)$, see eq. (41). In the integrating routine KORWAN we have implemented a broad series of the possible $D\left(x_{i}, s\right)$ 's, ranging from the third-order one exponentiated according to the Jadach-Ward prescription [16] to the second-order 
one exponentiated according to Kuraev-Fadin [17] prescription. Also a family of structure functions designed especially to mimic the YFS formulas of KORALW is provided in this routine. The actual formulas and discussion of the various abovementioned structure functions can be found in ref. [14].

The actual threefold integration in eq. (38) is done numerically with the help of the Gaussian-type integrating routines, originating mostly from the CERN program library. Changes of variable as well as factorizing out of most peaked part of the distribution are also performed. This part of the distribution is integrated analytically down to two-dimensional numerical integration.

\section{Structure of the Program}

In this section we provide the reader with a brief guide over the KORALW program. We will describe its main routines, libraries and library interfaces.

The KORALW version 1.02 program is written in FORTRAN77 and, in principle, its source code could be stored in a single big file ${ }^{8}$. However, we prefer to keep it divided into parts grouped in 8 UNIX subdirectories. Each of them contains a functionally related and well separated set of FORTRAN subroutines:

- korw - The core of the program, a low-level Monte Carlo generating the phase-space points according to the prescription of Sect. 2.7.

- model - A part of the code calculating the Born matrix element and higherorder corrections in the $\bar{\beta}_{i}$-functions.

- semian - The semi-analytical routine KORWAN with its subprograms.

- glib - An auxiliary library for KORALW, including glibk.f, the HBOOKlike histogramming package of ref. [18].

- interfaces - Interfaces to TAUOLA, PHOTOS and JETSET. The standard common block /HEPEVT/ is filled in there.

- tauola - the TAUOLA library.

- $k w l$ und - the PHOTOS and JETSET ${ }^{9}$ codes.

- demo - The demonstration decks, input data files and sample outputs of the program.

\footnotetext{
${ }^{8}$ Such a file can be automatically produced with the help of the script $k l e j$ which is located in the directory demo. This script creates also the new directory koralw-source.

${ }^{9}$ As sufficiently up-to-date version of this library is not available in the Computer Physics Communication library. We provide our CPC distribution file with the set of dummy routines named jestet74.f. Users of the KORALW program should replace this file by an appropriate link.
} 
In the UNIX distribution each directory is equipped with the makefile. Of particular interest to the user are the commands make demo and make demo 2 issued from the demo directory. These commands compile the program, run it with sample data files and compare outputs against the pre-prepared samples (for the moment only the Hewlett-Packard test outputs are provided).

\section{1 korww}

The main administering routine of the korw part (directory) is called koralw (mode, xpar,npar). Its first argument MODE frames the main loop of the generation. For MODE $=-1$ the initialization of the generator takes place. The input data parameters are transferred via the vectors xpar and npar. The actual initialization of the internal variables is done in the routine filexp. Here the user can find all the other parameters not defined in an input file. Any input parameter overwritten by filexp is then returned by koralw in xpar or npar. In that mode all necessary library initializations take place. MODE $=0$ is the main generation mode. Each call generates one Monte Carlo event. The calculation of $\bar{\beta}$-functions (subroutine betax) and, in unweighted mode, the rejection according to the best weight wtmod (see Table 6 for definition of $w$ tmod) take place in here. Also some internal monitoring is done here, including an important quantity - the value of the total cross section coming from the events with a total weight greater than the maximal weight set for rejection. Finally, MODE $=1$ provides all sorts of important information, with the total cross section and cross section over the maximal weight among them. Various final library printouts are generated here. In optional MODE $=2$ some further printouts are provided.

The heart of the generator is hidden in the routine karlud and its satellites. After initialization, the series of calls in karlud to various routines builds up a Monte Carlo event following all steps sketched in Sect. 2.7. First, the $v$ variable is generated by the routine vesk1w, which is a general-purpose one-dimensional event generator where the generated function is supplemented by the FORTRAN function rhosko. The crude $\sigma_{0}$ function is encoded in bornsc and the additional overall Born normalization is set in case of formula (32) in bnorl2. The YFSgen does all generation of the photonic degrees of freedom. A detailed description of this generation can be found in ref. [2], and will be skipped here. Next, having the $s^{\prime}$ fixed, the variables $s_{1}$ and $s_{2}$ are generated by the routine resms 2 . Generation

is done in the broader phase-space area defined by the function $\Theta_{S}^{\text {crude }}$ of eq. (26). The decay channels are chosen in the subroutine decay. The angular variables are generated by the routines cospro and cosdec. Finally, the routine kinew reconstructs the four-momenta of the final fermions, based on the earlier generated variables. kinew returns these four-momenta in the CMS frame. As a 'byproduct' kinew provides the effective beams and, stored in the common block bormom, the four-momenta of the final fermions transformed to the CMS' frame, i.e. the CMS frame of the effective beams. The common block bormom and the effective beams are 
provided exclusively for the calculation of the matrix element in subroutine wwborn. Call to wroorn, supplying the exact Born matrix element completes the single-event generation in karlud.

\section{2 model}

The central routine for the calculation of the Born matrix element is the function wwborn. It does the mass reduction, either directly or by calling kinew with the masses set to 0 . Next, it calls the wrprod and wdecay routines, calculating respectively the production and decay spin amplitudes of the $W$ bosons. Finally, the spin amplitudes are summed and squared by wrborn. An important subroutine used in the course of constructing production and decay amplitudes is called polvec. It calculates the polarization vectors of the $W$ boson in the rectangular basis.

The $\bar{\beta}$-functions are calculated in the routine betax. It returns separately all the LL components of the $\bar{\beta}_{0^{-}}, \bar{\beta}_{1^{-}}$and $\bar{\beta}_{2^{-}}$functions up to the second order.

\section{3 semian}

Semi-analytical calculations are interfaced by the subroutine korwan. This routine is not designed as a stand-alone routine, i.e. apart from the arguments the user has to fill a few commons required by korwan. Normally, these commons are all set by the filexp routine, called during the initialization mode of the koralw. In case korwan is used separately from koralw, a possible way to fill these commons could be by calling filexp explicitly with the xpar and npar arguments prepared like for koralw routine. Amongst korwan arguments the keymod key defines which version of structure functions is to be used in the calculation and keypre sets the precision level (levels 1, 2, 3 and 4 are implemented). korwan returns the total cross section and its absolute error. The structure functions are coded in function yfspho. The yfspho takes care also of the photonic singularities in the structure functions. The Born total cross section is calculated by the function xsmuta. The corresponding one-dimensional differential cross section is provided by the function d1muta. The actual integrand function is coded in d2muta. Appropriate changes of variables are done in xsmuta and d1muta.

\section{4 glib}

This auxiliary package of programs (also included in the BHLUMI program) does histogramming, book-keeping of the $\mathrm{MC}$ weight, plots histograms in the $\mathrm{HT}_{\mathrm{E}} \mathrm{X}$ format, stores and retrieves histograms into/from the disk. Quite often its entries have names and input/output parameters similar to the hbook and hplot packages from the CERN library. 


\section{5 interfaces}

The following three physics generation libraries are now interfaced to KORALW: (i) the $\tau$ decay library TAUOLA ${ }^{10}[19]$, (ii) the generator of radiative corrections used in $W$ and $\tau$ decays, PHOTOS [20], (iii) JETSET [21], used for hadronization of quark pairs produced in hadronic decays of $W$ 's. The form of the interfaces is constrained by the properties of the libraries. For the final-state particles TAUOLA and PHOTOS use a coding format of the common block advocated by the Particle Data Group PDG [22]. On the other hand JETSET has its own common block for the output, which is equally widespread. Fortunately, it is not a problem as JETSET includes a special routine that translates the common block of the PDG format into its own convention.

The natural solution is thus to write particles produced in the main part of KORALW into the common block of the PDG first. Later, if necessary, generate and update in the PDG common block the $\tau$-decay products. The $\tau^{ \pm}$leptons are assumed to be fully polarized along the flight direction. All this is done in the routine tohep with the help of routine filhep from the TAUOLA library in a rather simple way. The filhep routine is well tested. It is used in TAUOLA, KORALZ [3] and KORALB [23]. Subroutine filhep sets also logical flags to be used later by PHOTOS, which is finally called for the generation of bremsstrahlung in $W$ and $\tau$ decays.

In the routine tohad, hadronization of the quarks originating from $W$ decay can be performed. To this end, the contents of the (PDG) common block is translated into the JETSET proper common block with the help of its routine luhepc. Note that this is the first call to the JETSET library. Then, the position of quarks from $W^{-} / W^{+}$is found and call lujoin(2,ijoin) is executed twice. Finally parton shower and hadronization are performed with the help of call lushow and call luexec. See the description of JETSET [21] for more details.

The two interfaces are stored in files hepface.f and lundface. $f$ in the directory interfaces. In this directory there is also the tauola-photos-ini.f file, which contains initialization routines for the TAUOLA and PHOTOS libraries; see refs. $[19,20]$ for more details. Note that, as usual, TAUOLA initialization parameters, such as $\tau$ decay branching ratios have to be set to realistic values by the TAUOLA/KORALW user. The predefined boost routine tralo4 from the $\tau$ rest frame to the laboratory frame used by TAUOLA is also stored in this file.

\section{6 demo}

This directory contains two demonstration programs: demo.f and demo2.f. The first, shorter one is described in detail in Sect. 5.2. In both cases, the main program performs initialization. The input data are read from the data file and then koralw arguments - vectors npar and xpar are filled. After initialization, the events are gen-

\footnotetext{
${ }^{10}$ Version higher than 2.5 or that of the KORALW distribution should be used.
} 
erated. For demo2.f, histogramming is done with the help of the glibk package. The Monte Carlo events are decoded from the /hepevt/ common block [22]. At the end of the calculation, a final printout of the Monte Carlo results is provided. In demo. $f$ two calls to the semi-analytical routine korwan are performed; Born and secondorder ISR cross-sections are calculated. The data files pro.data.10k.key wgt.eq. 0 and pro.data.50k.keywgt.eq.1, together with the corresponding output files pro.output.10k.keywgt.eq. $0 . \mathrm{hp}$ and pro.output.50k.keyigt.eq.1.hp are located in the subdirectory 190gev. These output files were obtained on a HewlettPackard installation.

\section{How to Use the Program?}

In this section we discuss in some detail the input and output parameters of the KORALW, we show how to write a simple program calling KORALW and discuss the contents of the standard KORALW printout. We also do a similar description of the semi-analytical routine KORWAN.

\subsection{Input/Output Parameters}

The input parameters are sent to koralw via the vector arguments npar and xpar in MODE $=-1$. They are collected in Tables 1 and 2 . The additional internal-input parameters are set in MODE $=-1$ with the help of the routine filexp. They are collected in Table 3. In the generation mode (MODE $=0$ ) all the four-momenta of the event are provided in the standard common block/hepevt/. For those users who do not want to use the /hepevt/ common block, the alternative, internal commons /momset/ and /momdec/ can provide all four-momenta, as described in Tables 4 and 5 . In the case of weighted events, the weights available for the user in the common /wgtall/ are listed in Table 6 . To reconstruct the cross section out of the wtset weights one needs to multiply the crude cross section $(\operatorname{xpar}(30)$ in MODE $=1$ ) by the average of the wtcrud times chosen wtset. In Table 7 the post-generation $($ MODE $=1$ ) output parameters sent as arguments of koralw are collected. Finally, Table 8 summarizes the meanings of the arguments of the semi-analytical routine korwan.

\subsection{Simple User Program}

In this section we provide the user with a very simple demo program. It shows how to do initialization of the koralw routine, how to generate events and read its output. Also a call to the korwan routine is explicitly shown. The listing of the demo program is given in Appendix B and the actual file demo. $f$ is provided in the directory demo. 


\begin{tabular}{|c|c|}
\hline neter & Meaning \\
\hline npar & $\begin{array}{l}=1000 * \text { KeyCul }+100 * \text { KeyNLL+10*KeyFSR+Key ISR } \\
- \text { General radiation switch } \\
\text { KeyISR }=0 \text { Initial State Radiation is OFF, } \\
\text { KeyISR=1 Initial State Radiation is ON. } \\
\text { KeyFSR Final State Radiation switch, INACTIVE. } \\
\text { KeyNLL=0 sets to zero the Next-to Leading } \alpha / \pi \text { terms in YFS } \\
\text { form-factor, useful for comparisons, } \\
\text { KeyNLL=1 the } \alpha / \pi \text { terms are kept in YFS form-factor. } \\
\text { KeyCul Coulomb correction, INACTIVE. }\end{array}$ \\
\hline$n \operatorname{par}(2)=$ KeyPhy & 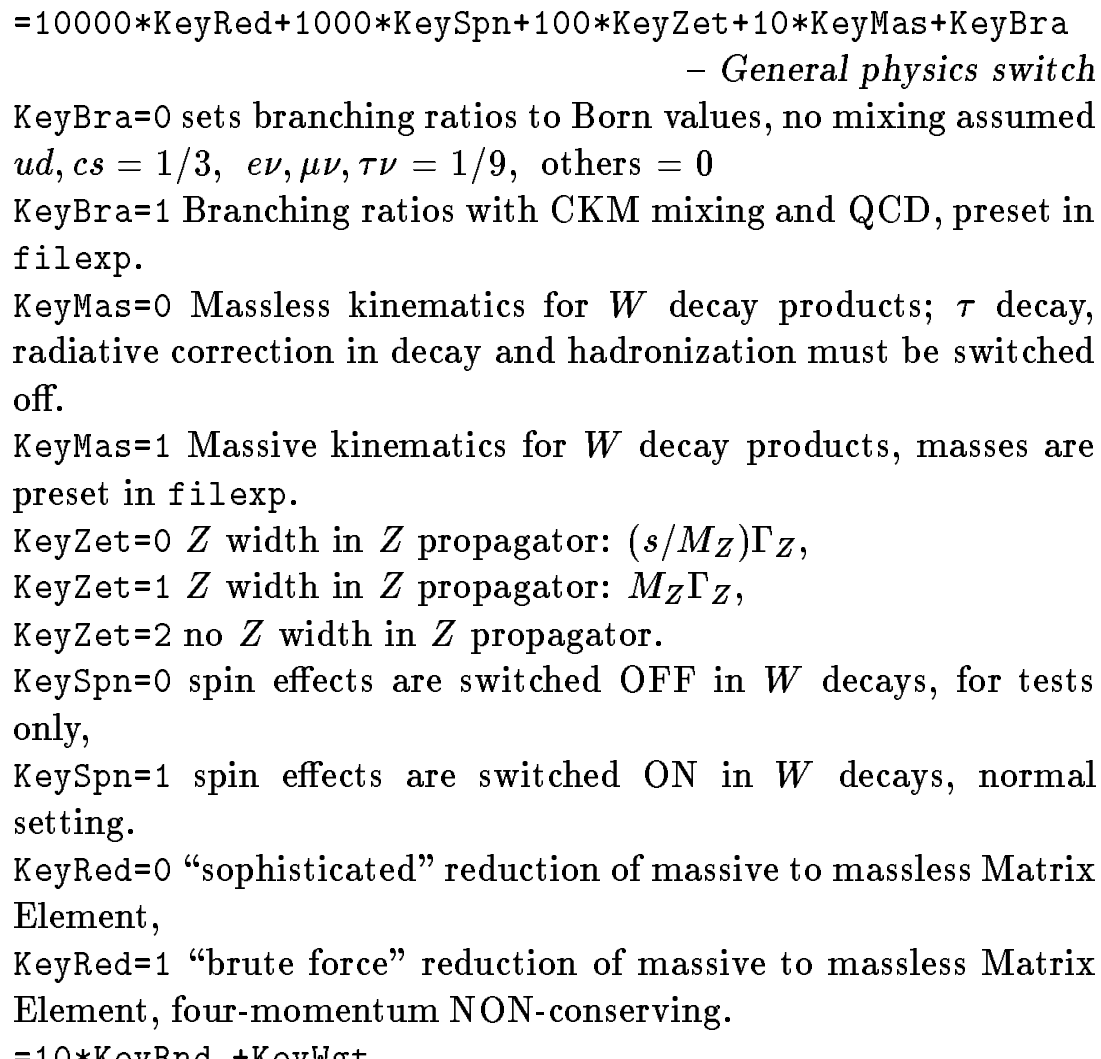 \\
\hline npar (4)=KeyMis & 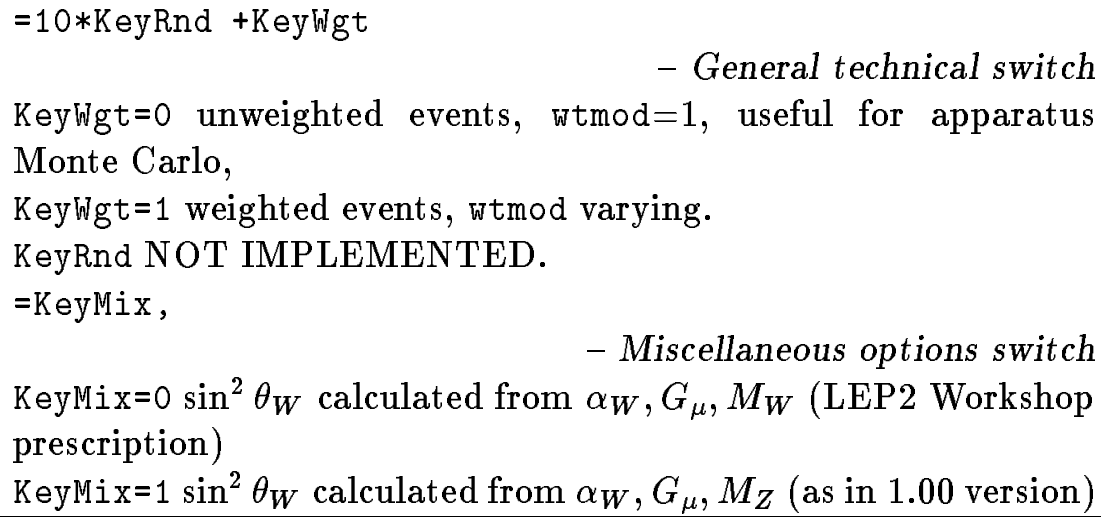 \\
\hline
\end{tabular}

Table 1: List of input parameters of the KORALW generator. 


\begin{tabular}{|c|c|}
\hline Parameter & Meaning \\
\hline$n p a r(5)=$ KeyDwm & Sets decay channel of $W^{-}$resonance \\
\hline npar $(6)=$ KeyDwp & Sets decay channel of $W^{+}$resonance \\
\hline & $0=$ all channels according to branching ratios \\
\hline & $1=u d, 2=c d, 3=u s, 4=c s, 5=u b, 6=c b, 7=e, 8=\mu, 9=\tau$ \\
\hline npar (7)=nout & Output unit number for the generator. \\
\hline$n \operatorname{par}(21)=j a k 1$ & For TAUOLA, sets $\tau$ of $W^{+}$decay channel \\
\hline$n \operatorname{par}(22)=\mathrm{jak} 2$ & $\begin{array}{l}\text { For TAUOLA, sets } \tau \text { of } W^{-} \text {decay channel } \\
\text { jak } 1, \text { jak2=-1 TAUOLA is OFF, } \\
\text { jak1, jak2=0 all channels, } \\
\text { jak1, jak2>0 single specific channel, see TAUOLA manual. }\end{array}$ \\
\hline npar (23) =itdkrc & $\begin{array}{l}\text { For TAUOLA, radiative corrections in leponic } \tau \text { decays switch, } \\
\text { itdkrc=1 corrections are ON, see TAUOLA manual, } \\
\text { itdkrc=0 corrections are OFF, see TAUOLA manual. }\end{array}$ \\
\hline npar (24)=ifphot & $\begin{array}{l}\text { PHOTOS, activation switch, } \\
\text { ifphot }=1 \text { PHOTOS is ON, } \\
\text { ifphot }=0 \text { PHOTOS is OFF. }\end{array}$ \\
\hline npar (25) =ifhadm & $W^{-}$hadronization activation switch (JETSET) \\
\hline npar (26) =ifhadp & $W^{+}$hadronization activation switch (JETSET) \\
\hline & ifhadm, ifhadp $=1$ hadronization is $\mathrm{ON}$ \\
\hline & ifhadm, ifhadp=0 hadronization is OFF. \\
\hline & For the moment ifhadm and ifhadp have to be equal! \\
\hline $\operatorname{xpar}(1)=\mathrm{cmsene}$ & $\sqrt{s}$, centre-of-mass (CMS) energy in GeV units. \\
\hline $\operatorname{xpar}(2)=g m u$ & $G_{F}$, Fermi constant. \\
\hline $\operatorname{xpar}(3)=\operatorname{alf} w$ in & $\begin{array}{l}1 / \alpha_{W} \text { inverse QED coupling constant at } M_{W} \text { scale, see Appendix } \\
\text { A. }\end{array}$ \\
\hline $\operatorname{xpar}(4)=\operatorname{amaz}$ & $M_{Z}$, mass of $Z$ boson in $\mathrm{GeV}$ \\
\hline $\operatorname{xpar}(5)=g a m m z$ & $\Gamma_{Z}$, width of $Z$ boson in $\mathrm{GeV}$ \\
\hline $\operatorname{xpar}(6)=\operatorname{amaw}$ & $M_{W}$, mass of $W$ boson in $\mathrm{GeV}$. \\
\hline $\operatorname{xpar}(7)=g \operatorname{amm} w$ & $\begin{array}{l}\Gamma_{W}, \text { width of } W \text { boson in } \mathrm{GeV} \text {, for }(\mathrm{gamm} \text { w }<0) \Gamma_{W} \text { is recalculated } \\
\text { from } G_{\mu} \text { and } M_{W} \text {. }\end{array}$ \\
\hline $\operatorname{xpar}(8)=\operatorname{vvmin}$ & Minimum $v$-variable (dimensionless), Infra-Red cut-off. \\
\hline $\operatorname{xpar}(9)=\operatorname{vvmax}$ & Maximum $v$-variable. \\
\hline $\operatorname{xpar}(10)=w \operatorname{tmax}$ & $\begin{array}{l}\text { Maximum weight for rejection, for wtmax }<0 \text { default settings of } \\
\text { koralw are used. }\end{array}$ \\
\hline
\end{tabular}

Table 2: List of input parameters of the KORALW generator. 


\begin{tabular}{|c|c|}
\hline Parameter & Meaning \\
\hline $\begin{array}{l}\text { alfinv } \\
\text { gpicob } \\
\text { amel } \\
\text { br (20) }\end{array}$ & $\begin{array}{l}1 / \alpha(0) \text { Inverse QED coupling constant at scale } Q^{2}=0 . \\
\text { Conversion from } \mathrm{GeV}^{-2} \text { to } \mathrm{pb} \text {. } \\
\text { Electron mass used by the bremsstrahlung generator. } \\
W \text { branching ratios, numbering of entries is: } \\
1=u d, 2=c d, 3=u s, 4=c s, 5=u b, 6=c b, 7=e, 8=\mu, 9=\tau ; \\
\text { br( } 7) \text { is always used for normalization and should not be set to } 0 \text {. } \\
\text { Masses of the } W \text { decay products; used entries are } \\
1=d, 2=u, 3=s, 4=c, 5=b \\
11=e, 12=\nu_{e}, 13=\mu, 14=\nu_{\mu}, 15=\tau, 16=\nu_{\tau} \text {; } \\
\text { masses of } \tau \text { and } \nu_{\tau} \text { have to be independently set to the same } \\
\text { numerical values in intialization of TAUOLA. }\end{array}$ \\
\hline
\end{tabular}

Table 3: List of input parameters of the KORALW generator set in the routine filexp.

\begin{tabular}{|c|c|}
\hline Parameter & Meaning \\
\hline$q \operatorname{qeff1(4)}$ & $\begin{array}{l}\text { Effective parameter for matrix element ( } e^{-} \text {effective beam, see } \\
\text { formula }(36)), C M S \text {. }\end{array}$ \\
\hline$q \in f f 2(4)$ & $\begin{array}{l}\text { Effective parameter for matrix element ( } e^{+} \text {effective beam, see } \\
\text { formula }(36)), C M S \text {. }\end{array}$ \\
\hline $\operatorname{sphum}(4)$ & Sum of four-momenta of IS photons, $C M S$. \\
\hline $\operatorname{sphot}(100,4)$ & Four-momenta of IS photons, $C M S$. \\
\hline nphot & Multiplicity of IS photons \\
\hline
\end{tabular}

Table 4: List of four-momenta in the internal common block /momset/ of the KO$R A L W$ generator.

\begin{tabular}{|l|l|}
\hline Parameter & Meaning \\
\hline \hline $\mathrm{q} 1(4)$ & Four-momentum of the $W^{-}$resonance, $C M S$. \\
$\mathrm{q} 2(4)$ & Four-momentum of the $W^{+}$resonance, $C M S$. \\
$\mathrm{p} 1(4)$ & Four-momentum of the fermion from $W^{-}$decay, $C M S$. \\
p2(4) & Four-momentum of the antifermion from $W^{-}$decay, $C M S$. \\
p3(4) & Four-momentum of the fermion from $W^{+}$decay, $C M S$. \\
p4(4) & Four-momentum of the antifermion from $W^{+}$decay, $C M S$. \\
\hline
\end{tabular}

Table 5: List of four-momenta in the internal common block /momdec/ of the KO$R A L W$ generator. 


\begin{tabular}{|c|c|}
\hline Parameter & Meaning \\
\hline wtcrud & Crude weight, necessary to build the total weight out of wtset. \\
\hline wtmod & Best weight \\
\hline & For Key ISR=0 wtmod=wtcrud*wtset (1), \\
\hline & For KeyISR=1 wtmod=wtcrud*wtset ( 3 ). \\
\hline $\operatorname{wtset}(100)$ & $\begin{array}{l}- \text { Born matrix el. with various } \bar{\beta} \text { contributions; } \\
\text { to get total weight must be multiplied by wtcrud. }\end{array}$ \\
\hline wtset (1) & Zero-order complete $\left(\bar{\beta}_{0}\right)$ \\
\hline wtset (2) & First-order complete $\left(\bar{\beta}_{0}+\bar{\beta}_{1}\right)$ \\
\hline wtset (3) & Second-order complete $\left(\bar{\beta}_{0}+\bar{\beta}_{1}+\bar{\beta}_{2}\right)$ \\
\hline wtset (20) & First-order $\bar{\beta}_{0}$ contribution alone \\
\hline wtset (21) & First-order $\bar{\beta}_{1}$ contribution alone \\
\hline wtset $(30)$ & Second-order $\bar{\beta}_{0}$ contribution alone \\
\hline wtset (31) & Second-order $\bar{\beta}_{1}$ contribution alone \\
\hline wtset (32) & Second-order $\bar{\beta}_{2}$ contribution alone \\
\hline
\end{tabular}

Table 6: List of output weights in the common block /wgtall/ of the KORALW generator.

\begin{tabular}{|c|c|}
\hline Parameter & Meaning \\
\hline npar (10)=nevtru & $\begin{array}{l}\text { For KeyWgt=0 number of accepted unweighted events, } \\
\text { For KeyWgt=1 number of generated weighted events. }\end{array}$ \\
\hline npar (11)=nevtot & $\begin{array}{l}\text { For KeyWgt=0 number of generated events before rejection, } \\
\text { For KeyWgt=1 npar (11)=npar (10) }\end{array}$ \\
\hline$x p a r(20)=x \operatorname{secpb}$ & Best cross section in $\mathrm{pb}$ \\
\hline & For KeyISR=0 Born cross section in pb, \\
\hline & For KeyISR=1 second-order exponentiated cross section in $\mathrm{pb}$. \\
\hline $\operatorname{xpar}(21)=\operatorname{errpb}$ & Error of total cross section $\mathrm{xsecpb}$, in $\mathrm{pb}$ \\
\hline $\operatorname{xpar}(30)=x \operatorname{crude}$ & Cross section in pb: \\
\hline & $\begin{array}{l}\text { For Keywgt }=0 \text { best cross section } \mathrm{xcrude} \text { nevtru/nevtot } * \text { wtmax, } \\
\text { For KeyWgt }=1 \text { crude cross section in } \mathrm{pb} \text {. }\end{array}$ \\
\hline$x p a r(31)=w \operatorname{tmax}$ & Maximal weight used for rejection. \\
\hline
\end{tabular}

Table 7: List of output parameters of the KORALW generator in Mode $=1$. 


\begin{tabular}{|c|c|}
\hline Parameter & Meaning \\
\hline & INPUT \\
\hline $\begin{array}{l}\text { svar } \\
\text { vvmin } \\
\text { vvmax } \\
\text { keymod }\end{array}$ & $\begin{array}{l}s, \text { CMS energy squared [GeV]. } \\
v_{\text {min }}, \text { minimal } v \text { variable, in most cases should be set to } 0 . \\
v_{\text {max }}, \text { maximal } v \text { variable. } \\
\text { Defines type of Structure Functions used for IS Radiation, } \\
\text { keymod }=0 \text { No ISR, Born, } \\
\text { keymod }=300 \text { Zero Order, YFS style, } \\
\text { keymod }=301 \text { First Order, YFS style, } \\
\text { keymod }=302 \text { Second Order, YFS style, } \\
\text { keymod }=303 \text { Third Order, YFS style, } \\
\text { keymod }=502 \text { Second Order, Gribov-Kuraev-Fadin style, } \\
\text { keymod }=310 \text { First Order YFS Beta0 only, } \\
\text { keymod }=311 \text { First Order YFS Beta1 only, } \\
\text { keymod }=320 \text { Second Order YFS Beta0 only, } \\
\text { keymod }=321 \text { Second Order YFS Beta1 only, } \\
\text { keymod }=322 \text { Second Order YFS Beta2 only, } \\
\text { keymod }<0 \text { as (-keymod) but multiplied by } v,(d \sigma / d \log v), \text { for tests } \\
\text { only. }\end{array}$ \\
\hline \multirow[t]{2}{*}{ keypre } & $\begin{array}{l}\text { Defines precision level of the computation. } \\
\text { For keymod }=0 \text { (No ISR, Born), in }\left(e \nu_{e}\right) \text { channel: } \\
\text { keypre }=1 \text { absolute error } 1 \times 10^{-5} \mathrm{pb}, \\
\text { keypre }=2 \text { absolute error } 1 \times 10^{-6} \mathrm{pb}, \\
\text { keypre }=3 \text { absolute error } 1 \times 10^{-7} \mathrm{pb}, \\
\text { For keymod }>0 \text { (ISR), in }\left(e \nu_{e}\right) \text { channel: } \\
\text { keypre }=1 \text { absolute error } 3 \times 10^{-5} \mathrm{pb} \\
\text { keypre }=2 \text { absolute error } 1 \times 10^{-5} \mathrm{pb} \\
\text { keypre }=3 \text { absolute error } 1 \times 10^{-6} \mathrm{pb} \\
\text { keypre }=4 \text { absolute error } 1 \times 10^{-7} \mathrm{pb} \text {. }\end{array}$ \\
\hline & OUTPUT \\
\hline $\begin{array}{l}\text { xsect } \\
\text { errabs }\end{array}$ & $\begin{array}{l}\text { Cross section in } \mathrm{pb} \text {. } \\
\text { Absolute error in } \mathrm{pb} \text {. }\end{array}$ \\
\hline
\end{tabular}

Table 8: List of arguments of the KORWAN routine. 
In this short demo we have explicitly called the LUND listing routine lulist. All the LUND part is marked by * ((( and * $)))$, and can be commented out if necessary.

Demo starts by initializing the histogramming package GLIBK used by KORALW. Also appropriate common cglib is set. Next, the vectors npar and xpar are loaded with parameters required to initialize the koralw routine. The koral w routine is then called in the initialization mode $(M O D E=-1)$. After that, koralw is ready to generate events $(M O D E=0)$. This takes place in the main loop of the program. Call to the koralw in the post generation mode $(M O D E=+1)$ provides output parameters in the vectors npar and xpar. Two of them, the total cross section and its error, are explicitly extracted and later on printed out.

Finally, the semi-analytical routine korwan is called for the second-order ISR total cross section and the result is printed out. An accuracy is set to a low value in order to make the execution time short. There is no separate initialization mode for korwan. One has to remember however, that all the necessary common blocks must be filled before calling the routine korwan. If korwan is used together with the koralw generator, the filexp routine called by koralw for $M O D E=-1$ takes care of this initialization. Otherwise the solution might be to call filexp explicitly, with the arguments xpar and npar prepared as for koralw. Important excerpts from the output of this program are listed in Appendix C.

Printout starts with the detailed specification of the actually used input parameters. Also logos of all the activated libraries (TAUOLA, PHOTOS, JETSET) are printed here. Next, a printout of the one full event is shown. The other events, 10 printed by default, are not shown. The chosen event is the second generated one. It is chosen because one of the $W$ bosons decays into $\tau \bar{\nu}$, and one can see TAUOLA doing the $\tau$ decay, whereas the other $W$ decays hadronically with the JETSET acting on the cascade.

The final reports of KORALW are collected in four windows: $V, A, B$ and $C$. Window $V$ provides technical information on the performance of the Monte Carlo sampler vesk1 $\mathrm{w}$, generating the vital $v$-distribution. The approximate integral of the $v$-function, as approximated by vesk $1 w$ is printed in line $V 4$, the reweighted (exact) value in line $V 5$ and the exact value by Gaussian integration in line $V 6$. Corresponding ratios follow in lines $V 7$ and $V 8$.

Window $A$ provides a technical internal report of the karlud routine, i.e. information on the crude distribution and the Born matrix element. Line $A 0$ gives the total number of the generated weighted events. Line $A 1$ shows the number of events with the negative weight wtcrud. This entry should be equal to zero. Line $A 2$ provides the value of the master crude distribution of eq. (32). The difference with line $V 6$ is in the YFS form-factor of eq. (10) which is not included in $V 6$. Line $A 3$ shows the average of the weight wtcrud, and the line $A 4$ - the corresponding "cross section" (no matrix element is included here - only the crude distribution formula). Lines $A 5-A 8$ repeat the information of lines $A 0-A 1, A 3-A 4$, but for the weight with the Born matrix element, i.e. wtcrud*wtset (1). Lines $A 9-A 11$ provide information 
on how many of the events have the weight wtcrud*wtset(1) over the wtmax, the maximal weight for rejection. Their contribution to the cross section in absolute units (picobarns) and relative to the Born cross section is listed in $A 10$ and $A 11$, respectively. Note that there are no $\bar{\beta}$-functions included in any of the printouts in Window $A$.

Window $B$ is devoted to the technical information on the first order $\bar{\beta}_{0^{-}}$and $\bar{\beta}_{1}$-functions. Entry $B 0$ is the number of negative $\bar{\beta}_{0}$ weights wtbe01. Entry $B 1$ is the average of the wtbe01 weight and line $B 2$ the average of the wtset $(20) *$ wtcrud weight. Entry $B 3$ is the contribution of $\bar{\beta}_{0}$ to the cross section (in picobarns). Entries $B 4-B 6$ are similar to the $B 0-B 1, B 3$ ones, but for the $\bar{\beta}_{1}$-function.

Window $C$ is the most important from the users point of view. It provides the total cross sections of the first and second orders $(C 2$ and $C 5)$, in picobarns, with absolute errors in picobarns. Also the average of the $\bar{\beta}_{0}+\bar{\beta}_{1}$ weight wtbe01+wtbe10 (up to the first order) is provided in line $C 1$, whereas the number of negative weights wtbe01+wtbe10 is given in line $C 0$. Similar entries for $\bar{\beta}_{0}+\bar{\beta}_{1}+\bar{\beta}_{2}$ the weight wtbe02+wtbe11+wtbe20 (up to the second order) are given in $C 4$ and $C 3$. A very important entry, the number of events with a total weight wtmod (wtmod is the Born weight for KeyISR=0 and the second order ISR weight for KeyISR=1) over wtmax, is shown in line $C 6$. The corresponding total cross section (in picobarns) from above the wtmax is printed in line $C 7$. Since wtmod is used in actual rejection for the unweighted events, the smallness of the $C 7$ entry is a good indication that the value of tmax is not chosen too low.

This completes the description of the output of KORALW. The remaining two entries shown in the demo output are produced by the demo program.

\section{Acknowledgements}

We thank Prof. B.F.L. Ward for stimulating discussions and comments. M.S. thanks E. Fernandez and M. Martinez for their hospitality and help during his stay in IFAE Barcelona, and B. Pietrzyk for his hospitality in LAPP Annecy, where the KORALW project was partly developed. Special thanks are due to M. Martinez for his help and support in the development of the project. The help of S. Orteu in writing the QCD interface in version 1.00 is acknowledged. Useful remarks of M. Gruenewald are also acknowledged. 


\section{A Matrix Element}

The full amplitude (accordingly to the normalization convention of ref. [24]) for the $W$-pair production and decay into channel $(k l)$ can be written as

$$
\begin{aligned}
\mathcal{M}_{k l} & =\mathcal{M}_{k l}\left(\Delta \sigma ; \sigma_{1}, \sigma_{2}, \sigma_{3}, \sigma_{4}\right) \\
& =\frac{1}{G_{W}\left(s_{1}\right)} \frac{1}{G_{W}\left(s_{2}\right)} \sum_{\lambda_{1}} \sum_{\lambda_{2}} \mathcal{M}_{1}\left(\Delta \sigma ; \lambda_{1}, \lambda_{2}\right) \mathcal{M}_{2}^{k}\left(\lambda_{1}, \sigma_{1}, \sigma_{2}\right) \mathcal{M}_{3}^{l}\left(\lambda_{2}, \sigma_{3}, \sigma_{4}\right),
\end{aligned}
$$

where $\Delta \sigma$ is the difference between $e^{-}$and $e^{+}$helicities ${ }^{11}, \lambda_{1}, \lambda_{2}$ are polarizations of the intermediate $W^{-}$and $W^{+}$, respectively, and $\sigma_{i}$ are helicities of the $W$-decay products.

The amplitude $\mathcal{M}_{1}$ is the polarization amplitude for the $W$-pair production in $e^{+} e^{-}$annihilation. For this amplitude we use the expression given in ref. $[9]^{12}$ :

$$
\begin{aligned}
\mathcal{M}_{1}\left(\Delta \sigma=-; \lambda_{1}, \lambda_{2}\right)=-e^{2} \sqrt{s}\{ & 2\left[\frac{1}{s}-\frac{\eta_{L}}{s-M_{Z}^{2}+i s \Gamma_{Z} / M_{Z}}\right] \\
\times & {\left[\left(Q_{2} \epsilon_{1}\right) F_{1}\left(\epsilon_{2}\right)-\left(Q_{1} \epsilon_{2}\right) F_{1}\left(\epsilon_{1}\right)-\left(\epsilon_{1} \epsilon_{2}\right) F_{1}\left(Q_{2}\right)\right] } \\
& \left.+\frac{\zeta_{L}}{\left(p_{1}-Q_{1}\right)^{2}} F_{3}\left(\epsilon_{2}, p_{1}-Q_{1}, \epsilon_{1}\right)\right\},
\end{aligned}
$$

where

$$
\begin{aligned}
& \eta_{\sigma}= \begin{cases}1-\frac{1}{2 \sin ^{2} \theta_{W}} & \text { for } \sigma=L, \\
1 & \text { for } \sigma=R,\end{cases} \\
& \zeta_{\sigma}= \begin{cases}\frac{1}{2 \sin ^{2} \theta_{W}} & \text { for } \sigma=L, \\
0 & \text { for } \sigma=R,\end{cases}
\end{aligned}
$$

and

$$
\begin{aligned}
G_{W}\left(s_{i}\right) & =s_{i}-M_{W}^{2}+i s_{i} \Gamma_{W} / M_{W}, \\
B W\left(s_{i}\right) & =\left|G_{W}\left(s_{i}\right)\right|^{2}
\end{aligned}
$$

$M_{W}$ and $\Gamma_{W}$ denote the mass and the width of the $W$ bosons, $Q_{1}, \epsilon_{1}$ and $Q_{2}, \epsilon_{2}$ are four-momenta and polarization vectors of $W^{-}$and $W^{+}$, respectively, while $s_{1}=Q_{1}^{2}$ and $s_{2}=Q_{2}^{2}$.

\footnotetext{
${ }^{11}$ Since the masses of the initial-state leptons are neglected in the matrix-element calculation $\left(m_{e}^{2} \ll s\right), \Delta \sigma=-$ or + only.

${ }^{12}$ Note that our $W W$-production amplitude differs from that in ref. [9] by a normalization factor and by the $Z$ boson propagator, where we have included the $Z$ width $\Gamma_{Z}$, and $M_{Z}$ denotes the $Z$ mass.
} 
The constant $e$ in eq. (43) denotes as usual the positron electric charge, for which in practical calculations we substitute

$$
e \longrightarrow e_{W}=\sqrt{4 \pi \alpha_{W}}
$$

whith $\alpha_{W}$ being the effective coupling constant at the $2 M_{W}$ scale related to the coupling constant $\alpha$ in the Thomson limit through the relation

$$
\alpha_{W}=\frac{\sqrt{2} G_{F} M_{W}^{2} \sin ^{2} \theta_{W}}{\pi}=\frac{\alpha}{1-\Delta r}
$$

where $G_{F}$ is the Fermi coupling constant, $\sin ^{2} \theta_{W}$ is the weak mixing parameter, and $\Delta r$ is a radiative correction to the relation between $W$ and $Z$ bosons masses. Using the effective coupling constant $\alpha_{W}$ instead of $\alpha$ in the Born distribution ${ }^{13}$ takes into account a major portion of radiative non-bremsstrahlung corrections [25].

The coefficients $F_{i}$ in eq. (43) are some functions of four-vectors contracted with Dirac matrices defined in ref. [9]. In order to write them down explicitly, let us introduce some auxiliary notation. For any $a$ being a four-vector in the Minkowski space, $a=\left(a^{0}, a^{1}, a^{2}, a^{3}\right)$, we define

$$
a_{ \pm}=a^{1} \pm i a^{2}, \quad \bar{a}_{ \pm}=a^{0} \pm a^{3} .
$$

Then the functions $F_{i}$ read

$$
\begin{aligned}
& F_{1}(a)=a_{-}, \\
& F_{3}(a, b, c)=\bar{a}_{+}\left(\bar{b}_{-} c_{-}-b_{-} \bar{c}_{-}\right)-a_{-}\left(\bar{b}_{+} \bar{c}_{-}-b_{+} c_{-}\right) .
\end{aligned}
$$

The amplitude for opposite values of $e^{+}$and $e^{-}$helicities, i.e. $\Delta \sigma=+$, can be obtained from eq. (43) by means of the interchange: $F_{i} \rightarrow-F_{i}^{*}$ and $L \rightarrow R$. The above expression for the production amplitude $\mathcal{M}_{1}$ has been derived within the spinor formalism, using the Weyl representation for Dirac matrices and a rectangular basis for polarization vectors (for more details see ref. [9]). It is given in the CMS' of the initial lepton beams with the $+z$-axis pointing in the $e^{-}$beam direction. Three vector boson couplings according to the Standard Model are only included, however extensions to the general type couplings at the $W W \gamma$ and $W W Z$ vertices, see e.g. ref. [8], are possible within the above formalism.

The amplitudes $\mathcal{M}_{2}^{k}$ and $\mathcal{M}_{3}^{l}$ describe respectively $W^{-}$and $W^{+}$decays into corresponding channels. Following ref. [8], the polarization amplitudes for $W^{-}$decay into massless fermions can be written in the form

$$
\mathcal{M}^{W^{-} \rightarrow f_{1} \bar{f}_{2}}\left(\lambda_{1}, \sigma_{1}, \sigma_{2}\right)=e g_{-}^{W f_{1} f_{2}} C \delta_{\sigma_{1},-} \delta_{\sigma_{2},+} 2 \sqrt{q_{1}^{0} q_{2}^{0}} S\left(q_{1}, \epsilon\left(Q_{1}, \lambda_{1}\right), q_{2}\right)_{--}^{-} .
$$

\footnotetext{
${ }^{13}$ We include $\alpha_{W}$ among the KORALW input parameters.
} 
The coupling factors for the $W$ decay are

$$
\begin{aligned}
& g_{-}^{W \nu e}=g_{-}^{W e \nu}=\frac{1}{\sqrt{2} \sin \theta_{W}} \\
& g_{-}^{W u_{i} d_{j}}=\left(g_{-}^{W d_{j} u_{i}}\right)^{*}=\frac{U_{i j}}{\sqrt{2} \sin \theta_{W}},
\end{aligned}
$$

where $\left(u_{1}, u_{2}, u_{3}\right)=(u, c, t),\left(d_{1}, d_{2}, d_{3}\right)=(d, s, b)$, and $U_{i j}$ denotes the CabibboKobayashi-Maskawa matrix elements. $C$ is the effective colour factor: $C=\sqrt{3}$ for quarks and $C=1$ for leptons. In practical calculations of the cross section the combination $C U_{i j}$ is absorbed into the $W$-decay branching ratios. For the electric charge $e$ we use again the substitution of eq. (47).

$S\left(q_{1}, \epsilon\left(Q_{1}, \lambda_{1}\right), q_{2}\right)_{--}^{-}$is a spinorial string function defined in Appendix $\mathrm{C}$ of ref. [8]. The polarization four-vector $\epsilon\left(Q_{1}, \lambda_{1}\right)$ of the $W^{-}$boson is given in the rectangular (real) basis, see Appendix $\mathrm{C}$ of ref. [8]. Using the notation of eq. (49), the spinorial string function corresponding to various final-state momenta configurations can be expressed as

1. $q_{1}^{3} \neq-q_{1}^{0}$ and $q_{2}^{3} \neq-q_{2}^{0}$ :

$$
S\left(q_{1}, \epsilon, q_{2}\right)_{--}^{-}=\frac{1}{2 \sqrt{q_{1}^{0} q_{2}^{0} \bar{q}_{1+} \bar{q}_{2+}}}\left[\bar{q}_{1+}\left(\bar{q}_{2+} \bar{\epsilon}_{-}-q_{2-} \epsilon_{+}\right)+q_{1+}\left(q_{2-} \bar{\epsilon}_{+}-\bar{q}_{2+} \epsilon_{-}\right)\right]
$$

2. $q_{1}^{3}=-q_{1}^{0}$ and $q_{2}^{3} \neq-q_{2}^{0}$ :

$$
S\left(q_{1}, \epsilon, q_{2}\right)_{--}^{-}=\frac{1}{\sqrt{2 q_{2}^{0} \bar{q}_{2+}}}\left(q_{2-} \bar{\epsilon}_{+}-\bar{q}_{2+} \epsilon_{-}\right),
$$

3. $q_{1}^{3} \neq-q_{1}^{0}$ and $q_{2}^{3}=-q_{2}^{0}$ :

$$
S\left(q_{1}, \epsilon, q_{2}\right)_{--}^{-}=\frac{1}{\sqrt{2 q_{1}^{0} \bar{q}_{1+}}}\left(\bar{q}_{1+} \epsilon_{+}-q_{1+} \bar{\epsilon}_{+}\right),
$$

4. $q_{1}^{3}=-q_{1}^{0}$ and $q_{2}^{3}=-q_{2}^{0}$ :

$$
S\left(q_{1}, \epsilon, q_{2}\right)_{--}^{-}=\bar{\epsilon}_{+}
$$

For the $W^{+}$decay one merely needs to substitute $\epsilon\left(Q_{1}, \lambda_{1}\right) \rightarrow \epsilon\left(Q_{2}, \lambda_{2}\right)$. The formulae for the decay amplitudes are given in the massless fermion limit, which is justified by the fact that all $W$-decay products are relatively light when compared to the $W$-boson mass. In such a case expressions for the polarization amplitudes are particularly simple and fast in numerical evaluations. However, including the fermion masses, or any other modifications, in the above amplitudes does not pose a major problem and can be done in the future, if necessary. Care must be taken 
of the normalization and relative amplitude phases, especially if they originate from calculations based on different conventions.

The polarization-averaged Born cross section for the $W$-pair production and decay into the channel $(k l)$ can then be obtained from the formula:

$$
d \sigma_{k l}=\frac{1}{2 s} \frac{1}{4} \sum\left|\mathcal{M}_{k l}\right|^{2} d \Phi_{4}
$$

with $d \Phi_{4}$ being the invariant four-body phase space

$$
d \Phi_{4}=(2 \pi)^{4} \delta^{(4)}\left(p_{1}+p_{2}-\sum_{i=1}^{4} q_{i}\right) \prod_{i=1}^{4} \frac{d^{3} q_{i}}{(2 \pi)^{3} 2 q_{i}^{0}}
$$

and the sum $\sum\left|\mathcal{M}_{k l}\right|^{2}$ running over all initial- and final-states polarizations. The factor $\frac{1}{4}$ in formula (58) stands for the initial-state spin average. 


\section{B Listing of the Simple Program}

PROGRAM MAIII

IMPLICIT DOUBLE PRECISION $(\mathrm{A}-\mathrm{H}, \mathrm{O}-\mathrm{Z})$

DIMENSION XPAR (100), IIPAR (100)

* (l(C LUND stuff

COMMON / LUDAT1 / MSTU, PARU, MST J, PARJ

REAL PARU (200), PARJ (200)

INTEGER MSTU(200), MSTJ (200)

*))) end LUND stuff

* For internal histogramming package GLIBK

COMMOII / CGLIB / BLIBK (20000)

SAVE / CGLIB /

SAVE

IIOUT $=16$

OPEII (IIOUT , file=' pro. output')

CALL GLIMIT (20000)

CALL GOUTPU(NOUT)

* End of initialization of internal histogramming package GLIBK

* ( ( $\quad L U N D$ stuff mstu(11) $=$ nout

*)))) end LUND stuff

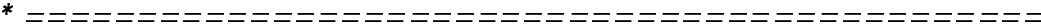

* Prepare the input parameters for KORALW

$\operatorname{IPAR}(1)=1 \quad !$ KeyRad $=1000 * \mathrm{KeyCu}+100 * \mathrm{Key} \mathbb{N L L}+10 * \mathrm{KeyFSR}+\mathrm{Key}$ ISR

$\operatorname{MPAR}(2)=1011 ! \mathrm{KeyPhy}=10000 *$ KeyRed $+1000 *$ Key Spn $+100 *$ KeyZet

$\operatorname{IIPAR}(3)=10 \quad !$ KeyTek $=10 *$ KeyRnd + Keyhgt

$+10 *$ KeyMas+KeyBra

$\operatorname{MPAR}(4)=0 \quad !$ KeyMis $=$ KeyMix

$\operatorname{MPAR}(5)=0 \quad !$ KEYDWM $\mathrm{W}-$ decay: $7=(\mathrm{ev}), 0=\mathrm{all} \mathrm{ch}$

$\operatorname{IIPAR}(6)=0 \quad ! \operatorname{KEYDWP} W+$ decay $: 7=(\mathrm{ev}), 0=\mathrm{all} \mathrm{ch}$.

$\operatorname{IIPAR}(7)=\mathbb{N}$ OUT $! \mathbb{I N O U T} \quad$ Output unit number, for $\mathbb{N o u t}$. LE. 0 , Ilout=16

$\operatorname{IIPAR}(21)=0 \quad !$ JAK1 Decay mode tau+

$\operatorname{IIPAR}(22)=0 \quad$ JAK2 Decay mode tau-

$\operatorname{IIPAR}(23)=1 \quad$ ITDKRC Bremsstrahlung stitch in Tauola

$\operatorname{IIPAR}(24)=1 \quad$ IFPHOT PHOTOS suitch

$\operatorname{IIPAR}(25)=1$ ! IFHADM Hadronisation W-

$\operatorname{IIPAR}(26)=1 \quad$ I IFHADP Hadronisation W+IFHADP

$\operatorname{XPAR}(1)=180 \mathrm{DO} \quad !$ CMSENE CMS total energy

XPAR (2) $=1.16639 D-5$ ! GFERMI Fermi Constant

XPAR (3) $=128.07 D 0$ ! ALFWIII alpha QED at WW tresh. scale (inverse)

$\mathrm{XPAR}(4)=91.1888 \mathrm{~d} 0 \quad$ ! AMAZ $\mathrm{Z}$ mass

$\operatorname{XPAR}(5)=2.4974 \mathrm{dO} \quad !$ GAMMZ $\mathrm{Z}$ ridth

$\operatorname{XPAR}(6)=80.230 \mathrm{~d} 0 \quad !$ AMAW W mass

$\operatorname{XPAR}(7)=-2.03 \mathrm{~d} 0 \quad !$ GAMMb W Hith,

$! \quad$ For GAMMH<0 RECALCULATED inside program

$\operatorname{XPAR}(8)=1 \mathrm{D}-6$

! VVMIII Photon spectrum parameter

! VVMAX Photon spectrum parameter

$X P A R(9)=0.99 D 0$

$\operatorname{XPAR}(10)=-1 \mathrm{DO}$

! WTMAX max reight for reject.

wTMA $<0=$ default setting

input for korwan

* End of setting parameters

* KORALW initialisation

CALL KORALW $(-1$, XPAR, IPPAR

* end KORALW initialisation

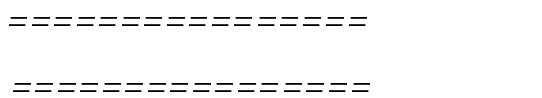


* KORALW main loop

D0 $100 \mathrm{I}=1,1000$

CALL KORALW ( 0 , XPAR, IIPAR)

* ( ( ( LUND stuff IF (I.LE.10) CALL lulist(2)

*)))) end LUND stuff

100 COITTIUUE

* End KORALW main loop

* final printouts of KORALW ニニニニニニニニニニニニニニニニ CALL KORALW ( 1 , XPAR, IPAR)

* some output parameters of KORALW

XSECT $=\operatorname{xpar}(20)$

ERSECT $=x p a r(21)$

* end final printouts of KORALW

* SemiA nalytical O(alf2)exp. , KORWAN keypho $=302$

keyacc $=1$

call korнan (cmsene**2, Od0,1d0, keypho, keyacc, Xtot2e, errabs)

* End SemiAnalytical O(alf2)exp. , KORWAN

* Final printouts of demo

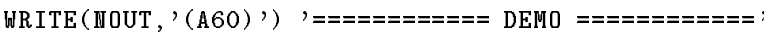
WRITE (HOUT , *)

WRITE(HOUT ,' (1X,F17.8, 4H +-, F11.8, $\left.1 \mathrm{X}, \mathrm{A} 30)^{\prime}\right)$ )

$\$ \quad$ xsect,ersect, 'MC Best, XPAR, KORALW,

WRITE (NOUT , *)

WRITE(NOUT, ' ( $\left.1 \mathrm{X}, \mathrm{F} 17.8,4 \mathrm{H}+-, \mathrm{F} 11.8,1 \mathrm{X}, \mathrm{A} 30)^{\prime}\right)$ )

$\$ \quad X$ tot $2 e$,errabs, 'SemiAnal 0(alf2)exp.LL, KORWAII'

WRITE (NOUT , *)

WRITE (IIOUT , '(A60)') '========== End DEMO $=========$,

END 


\section{Output of the Simple Program}

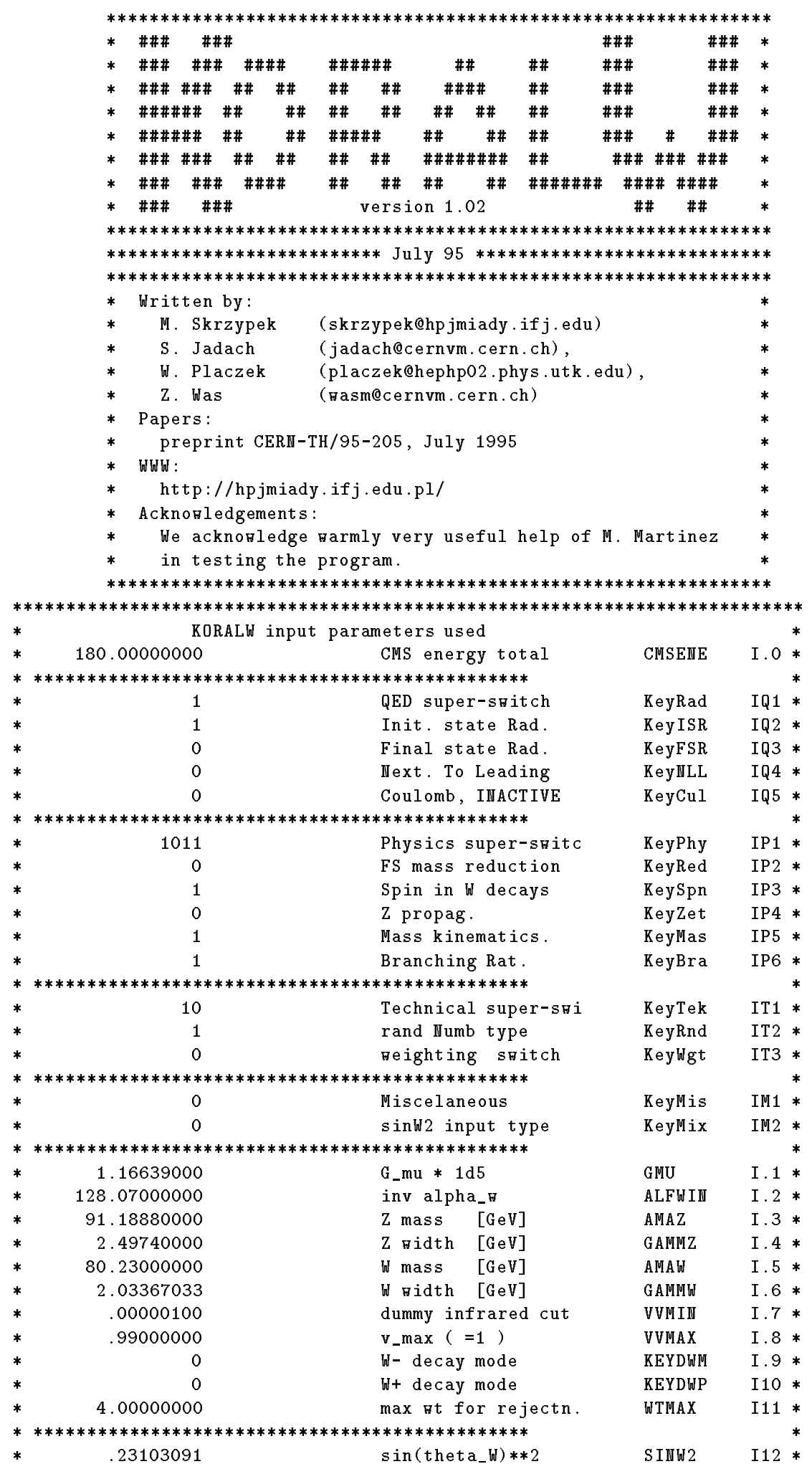




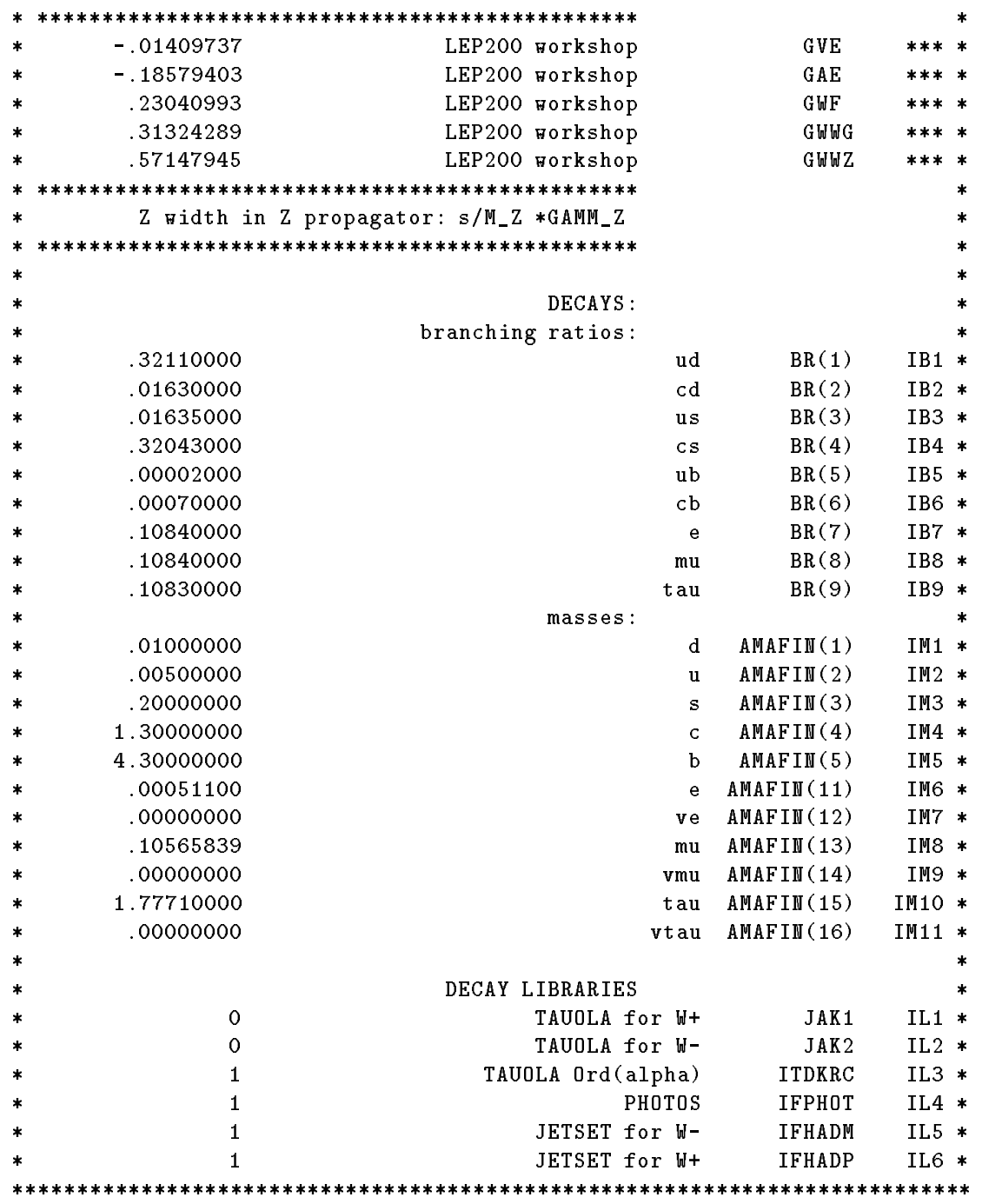

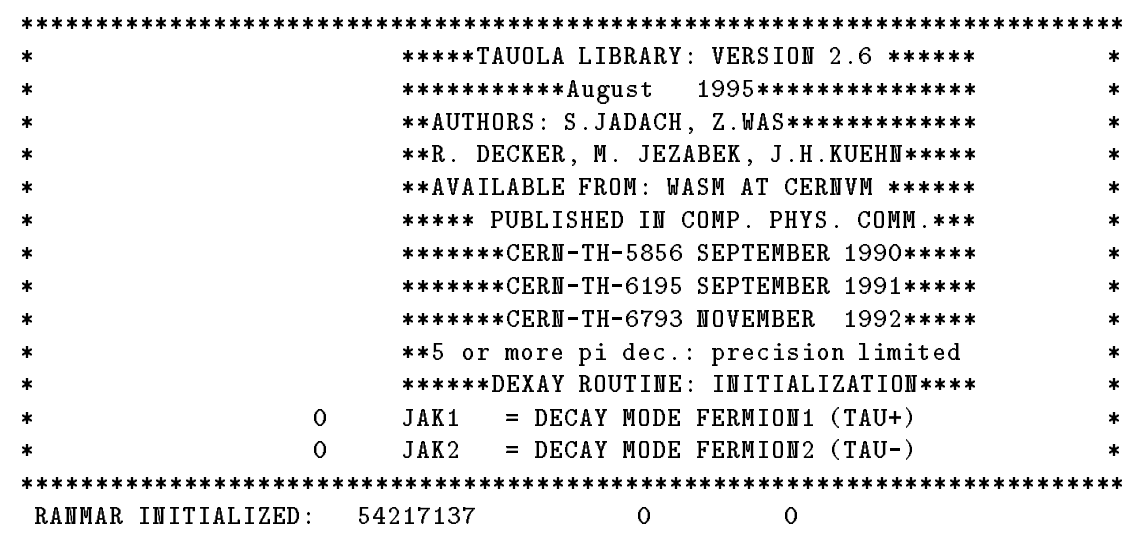




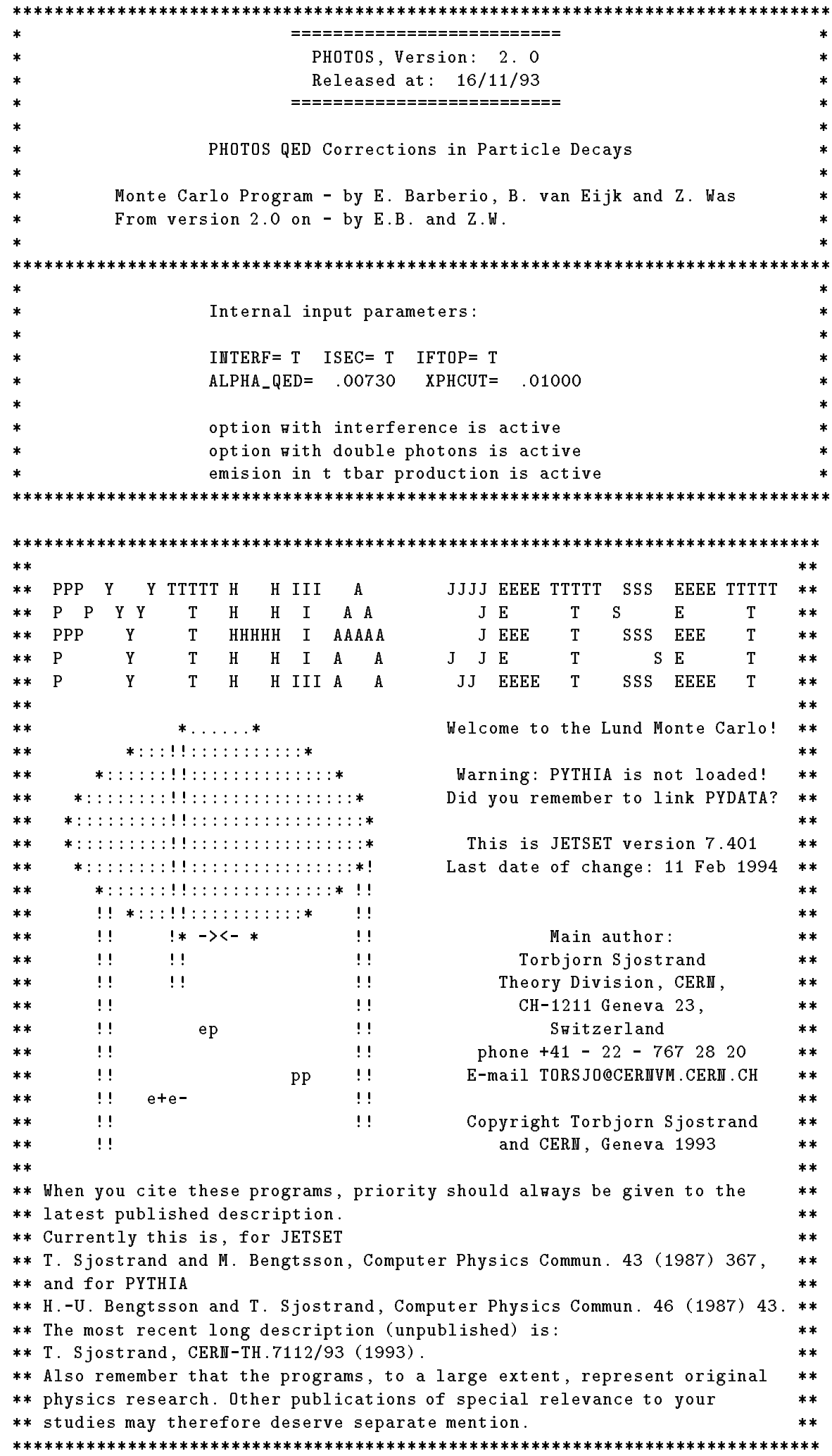


first event skipped

\begin{tabular}{|c|c|c|c|c|c|}
\hline & $p(1)$ & $p(2)$ & $p(3)$ & $p(4)$ & pdg-code \\
\hline PHO & .00012717 & -.00127282 & -.00238594 & .00270720 & 22 \\
\hline PHO & .00000061 & .00000032 & -.00017966 & .00017966 & 22 \\
\hline $\mathrm{W}-$ & -27.73493798 & -10.83832552 & 26.10287894 & 90.69685708 & -24 \\
\hline $\mathrm{W}+$ & 27.73481021 & 10.83959801 & -26.10031334 & 89.30025606 & 24 \\
\hline $\mathrm{p} 1$ & -48.01722190 & -18.46743658 & -6.14744728 & 51.84254050 & 15 \\
\hline $\mathrm{p} 2$ & 20.28228392 & 7.62911106 & 32.25032621 & 38.85431659 & -16 \\
\hline p3 & -13.00605304 & -9.29593565 & 19.27808861 & 25.07800900 & 4 \\
\hline $\mathrm{p} 4$ & 40.74086324 & 20.13553366 & -45.37840195 & 64.22224706 & -3 \\
\hline sum & .00000000 & .00000000 & .00000000 & 180.00000000 & 0 \\
\hline
\end{tabular}

Event listing (standard)

\begin{tabular}{|c|c|c|c|c|c|c|c|c|c|c|c|c|c|c|c|}
\hline I & particle/jet & $K(I, 1)$ & $K(I, 2)$ & $K(I, 3)$ & & $K(I$ & ,4) & & $K(I$ & ,5) & $P(I, 1)$ & $P(I, 2)$ & $P(I, 3)$ & $P(I, 4)$ & $P(I, 5)$ \\
\hline 1 & $!_{\theta-1}$ & 21 & 11 & 0 & & & 3 & & & 6 & .00000 & .00000 & 90.00000 & 90.00000 & .00051 \\
\hline 2 & $! \theta+!$ & 21 & -11 & 0 & & & 3 & & & 6 & .00000 & .00000 & -90.00000 & 90.00000 & .00051 \\
\hline 3 & $(\mathfrak{W}-)$ & 11 & -24 & 1 & & & 7 & & & 8 & -27.73494 & -10.83833 & 26.10288 & 90.69685 & 81.59573 \\
\hline 4 & $(\mathrm{~h}+)$ & 11 & 24 & 1 & & & 9 & & & 10 & 27.73481 & 10.83960 & -26.10031 & 89.30025 & 80.04119 \\
\hline 5 & gamma & 1 & 22 & 1 & & & 0 & & & 0 & .00013 & -.00127 & -.00239 & .00271 & .00000 \\
\hline 6 & gamma & 1 & 22 & 1 & & & 0 & & & 0 & .00000 & .00000 & -.00018 & .00018 & .00000 \\
\hline 7 & $\left(\tan ^{-}\right)$ & 11 & 15 & 3 & & & 11 & & & 12 & -48.01722 & -18.46744 & -6.14745 & 51.84254 & 1.77710 \\
\hline 8 & nu_tau" & 1 & -16 & 3 & & & 0 & & & 0 & 20.28228 & 7.62911 & 32.25033 & 38.85432 & .00000 \\
\hline 9 & (c) & 14 & 4 & 4 & 3 & 10 & 17 & 0 & 0 & 17 & -13.00605 & -9.29594 & 19.27809 & 25.07801 & 1.30000 \\
\hline 10 & $\left(s^{*}\right)$ & 14 & -3 & 4 & 0 & 0 & 18 & 3 & 9 & 18 & 40.74086 & 20.13553 & -45.37840 & 64.22224 & .20000 \\
\hline 11 & nu_tau & 1 & 16 & 7 & & & 0 & & & 0 & -12.76560 & -4.97430 & -1.42791 & 13.77473 & .00063 \\
\hline 12 & $(\mathfrak{W}-)$ & 11 & -24 & 7 & & & 13 & & & 15 & -35.25161 & -13.49314 & -4.71954 & 38.06780 & 1.46381 \\
\hline 13 & (eta) & 11 & 221 & 12 & & & 33 & & & 34 & -21.84054 & -8.43183 & -3.26327 & 23.64434 & .54880 \\
\hline 14 & $\mathrm{pi}^{-}$ & 1 & -211 & 12 & & & 0 & & & 0 & -9.94241 & -3.51432 & -.93589 & 10.58760 & .13957 \\
\hline 15 & (pio) & 11 & 111 & 12 & & & 35 & & & 36 & -3.46867 & -1.54698 & -.52038 & 3.83586 & .13496 \\
\hline 16 & (CHshower) & 11 & 94 & 9 & & & 17 & & & 18 & 27.73481 & 10.83960 & -26.10031 & 89.30025 & 80.04119 \\
\hline 17 & (c) & 14 & 4 & 16 & 3 & 9 & 20 & 0 & 9 & 19 & -12.77663 & -9.17593 & 19.01150 & 25.33461 & 5.74152 \\
\hline 18 & $\left(\mathrm{~s}^{-}\right)$ & 14 & -3 & 16 & 0 & 10 & 21 & 3 & 10 & 22 & 40.51144 & 20.01553 & -45.11182 & 63.96565 & 3.83799 \\
\hline 19 & $(c)$ & 14 & 4 & 17 & 3 & 20 & 24 & 0 & 17 & 23 & -12.88282 & -9.11275 & 19.03638 & 25.20857 & 4.90701 \\
\hline 20 & $(g)$ & 13 & 21 & 17 & 2 & 17 & 0 & 2 & 19 & 0 & .10619 & -.06318 & -.02488 & .12604 & .00000 \\
\hline 21 & $\left(\mathrm{~s}^{*}\right)$ & 13 & -3 & 18 & 0 & 18 & 0 & 2 & 22 & 0 & 20.74255 & 10.22071 & -20.38377 & 30.82619 & .19900 \\
\hline 22 & $(g)$ & 13 & 21 & 18 & 2 & 21 & 0 & 2 & 18 & 0 & 19.76889 & 9.79482 & -24.72804 & 33.13946 & .00000 \\
\hline 23 & (c) & 14 & 4 & 19 & 3 & 24 & 26 & 0 & 19 & 25 & -13.05451 & -9.12877 & 18.90506 & 24.99181 & 3.66534 \\
\hline 24 & (g) & 13 & 21 & 19 & 2 & 19 & 0 & 2 & 23 & 0 & .17170 & .01602 & .13133 & .21676 & .00000 \\
\hline 25 & (c) & 13 & 4 & 23 & 2 & 26 & 0 & 0 & 23 & 0 & -12.67742 & -9.43382 & 18.29591 & 24.21315 & 1.35000 \\
\hline 26 & $(\mathrm{~g})$ & 13 & 21 & 23 & 2 & 23 & 0 & 2 & 25 & 0 & -.37710 & .30505 & .60915 & .77866 & .00000 \\
\hline 27 & $\left(\mathrm{~s}^{*}\right)$ & 12 & -3 & 21 & & & 37 & & & 37 & 20.74255 & 10.22071 & -20.38377 & 30.82619 & .19900 \\
\hline 28 & $(\mathrm{~g})$ & 12 & 21 & 22 & & & 37 & & & 37 & 19.76889 & 9.79482 & -24.72804 & 33.13946 & .00000 \\
\hline 29 & (g) & 12 & 21 & 20 & & & 37 & & & 37 & .10619 & -.06318 & -.02488 & .12604 & .00000 \\
\hline 30 & (g) & 12 & 21 & 24 & & & 37 & & & 37 & .17170 & .01602 & .13133 & .21676 & .00000 \\
\hline 31 & (g) & 12 & 21 & 26 & & & 37 & & & 37 & -.37710 & .30505 & .60915 & .77866 & .00000 \\
\hline 32 & (c) & 11 & 4 & 25 & & & 37 & & & 37 & -12.67742 & -9.43382 & 18.29591 & 24.21315 & 1.35000 \\
\hline 33 & gamma & 1 & 22 & 13 & & & 0 & & & 0 & -18.61424 & -7.01143 & -2.66166 & 20.06825 & .00000 \\
\hline 34 & gamma & 1 & 22 & 13 & & & 0 & & & 0 & -3.22630 & -1.42040 & -.60160 & 3.57609 & .00000 \\
\hline 35 & gamma & 1 & 22 & 15 & & & 0 & & & 0 & -1.95753 & -.85912 & -.22651 & 2.14972 & .00000 \\
\hline 36 & gamma & 1 & 22 & 15 & & & 0 & & & 0 & -1.51114 & -.68787 & -.29387 & 1.68614 & .00000 \\
\hline 37 & (string) & 11 & 92 & 27 & & & 38 & & & 49 & 27.73481 & 10.83960 & -26.10031 & 89.30025 & 80.04120 \\
\hline 38 & (KO) & 11 & 311 & 37 & & & 50 & & & 50 & 7.68898 & 3.59828 & -7.98609 & 11.66591 & .49770 \\
\hline 39 & $n=0$ & 1 & -2112 & 37 & & & 0 & & & 0 & 10.02494 & 5.13412 & -11.08608 & 15.83170 & .93960 \\
\hline 40 & (LambdaO) & 11 & 3122 & 37 & & & 51 & & & 52 & 9.08600 & 4.49971 & -9.40533 & 13.87471 & 1.11560 \\
\hline 41 & $(\mathrm{~K} *+)$ & 11 & 323 & 37 & & & 53 & & & 54 & 9.80283 & 4.73202 & -12.05487 & 16.26360 & .83495 \\
\hline 42 & (pio) & 11 & 111 & 37 & & & 55 & & & 56 & 1.85638 & .68310 & -1.20573 & 2.32051 & .13500 \\
\hline 43 & (omega) & 11 & 223 & 37 & & & 57 & & & 59 & .28113 & 1.01223 & -.69439 & 1. 48162 & .78063 \\
\hline 44 & $($ rhoo $)$ & 11 & 113 & 37 & & & 60 & & & 61 & 1.02011 & -.12029 & -1.46830 & 1.95576 & .78357 \\
\hline 45 & (omega) & 11 & 223 & 37 & & & 62 & & & 64 & .06061 & .12238 & -.07067 & .78798 & .77283 \\
\hline 46 & $\mathrm{pi}^{-}$ & 1 & -211 & 37 & & & 0 & & & 0 & .34901 & .27016 & .23146 & .51755 & .13960 \\
\hline 47 & $($ rhot) & 11 & 213 & 37 & & & 65 & & & 66 & -.27300 & .22344 & -.26637 & .85588 & .73289 \\
\hline 48 & $\mathrm{~K}-$ & 1 & -321 & 37 & & & 0 & & & 0 & -5.16155 & -4.56596 & 7.96976 & 10.54752 & .49360 \\
\hline 49 & $\left(D_{-} s^{+}\right)$ & 11 & 431 & 37 & & & 67 & & & 69 & -7.00065 & -4.74960 & 9.93631 & 13.19751 & 1.96880 \\
\hline 50 & (K_SO) & 11 & 310 & 38 & & & 70 & & & 71 & 7.68898 & 3.59828 & -7.98609 & 11.66591 & .49770 \\
\hline 51 & $\mathrm{p}^{+}$ & 1 & 2212 & 40 & & & 0 & & & 0 & 7.59320 & 3.85463 & -7.97283 & 11.70305 & .93830 \\
\hline 52 & $\mathrm{pi}^{-}$ & 1 & -211 & 40 & & & 0 & & & 0 & 1.49280 & .64508 & -1.43249 & 2.17166 & .13960 \\
\hline 53 & $\mathrm{~K}+$ & 1 & 321 & 41 & & & 0 & & & 0 & 7.75578 & 3.50193 & -9.50857 & 12.76998 & .49360 \\
\hline 54 & (pio) & 11 & 111 & 41 & & & 72 & & & 73 & 2.04705 & 1.23009 & -2.54630 & 3.49362 & .13500 \\
\hline 55 & gamma & 1 & 22 & 42 & & & 0 & & & 0 & .81035 & .36544 & -.56757 & 1.05468 & .00000 \\
\hline 56 & gamma & 1 & 22 & 42 & & & 0 & & & 0 & 1.04602 & .31766 & -.63816 & 1.26583 & .00000 \\
\hline 57 & $\mathrm{pi+}^{\mathrm{i}}$ & 1 & 211 & 43 & & & 0 & & & 0 & .30309 & .55123 & -.32158 & .72015 & .13960 \\
\hline 58 & $\mathrm{pi}^{-}$ & 1 & -211 & 43 & & & 0 & & & 0 & .11908 & .14578 & .00185 & .23436 & .13960 \\
\hline 59 & (pio) & 11 & 111 & 43 & & & 74 & & & 75 & -.14104 & .31522 & -.37465 & .52711 & .13500 \\
\hline
\end{tabular}




\begin{tabular}{|c|c|c|c|c|c|c|c|c|c|c|c|}
\hline 60 & $\mathrm{pi}^{-}$ & 1 & -211 & 44 & 0 & 0 & .15507 & -.17319 & -.82227 & .86583 & .13960 \\
\hline 61 & $\mathrm{pi}^{\mathrm{i}}$ & 1 & 211 & 44 & 0 & 0 & .86505 & .05290 & -.64603 & 1.08993 & .13960 \\
\hline 62 & $\mathrm{pi}^{-}$ & 1 & -211 & 45 & 0 & 0 & .04608 & .05101 & -.20498 & .25735 & .13960 \\
\hline 63 & $\mathrm{pi}^{+}$ & 1 & 211 & 45 & 0 & 0 & .16278 & -.06672 & .17382 & .28399 & .13960 \\
\hline 64 & (pio) & 11 & 111 & 45 & 76 & 77 & -.14824 & .13809 & -.03951 & .24664 & .13500 \\
\hline 65 & pi+ & 1 & 211 & 47 & 0 & 0 & -.09925 & .45889 & -.18436 & .52336 & .13960 \\
\hline 66 & (pio) & 11 & 111 & 47 & 78 & 79 & -.17375 & -.23544 & -.08202 & .33252 & .13500 \\
\hline 67 & (pio) & 11 & 111 & 49 & 80 & 81 & -3.98092 & -2.11017 & 5.79007 & 7.33783 & .13500 \\
\hline 68 & (omega) & 11 & 223 & 49 & 82 & 83 & -2.44734 & -2.08769 & 3.10957 & 4.54583 & .80448 \\
\hline 69 & $\mathrm{pi}^{\mathrm{+}}$ & 1 & 211 & 49 & 0 & 0 & -.57239 & -.55174 & 1.03667 & 1.31386 & .13960 \\
\hline 70 & $\mathrm{pi}^{-}$ & 1 & -211 & 50 & 0 & 0 & 5.87131 & 2.83530 & -6.31194 & 9.07586 & .13960 \\
\hline 71 & $\mathrm{pit}$ & 1 & 211 & 50 & 0 & 0 & 1.81767 & .76298 & -1.67416 & 2.59005 & .13960 \\
\hline 72 & gamma & 1 & 22 & 54 & 0 & 0 & 1.13028 & .66950 & -1.49755 & 1.99209 & .00000 \\
\hline 73 & gamma & 1 & 22 & 54 & 0 & 0 & .91677 & .56059 & -1.04875 & 1.50154 & .00000 \\
\hline 74 & gamma & 1 & 22 & 59 & 0 & 0 & .00686 & .02864 & -.09762 & .10196 & .00000 \\
\hline 75 & gamma & 1 & 22 & 59 & 0 & 0 & -.14790 & .28657 & -.27703 & .42514 & .00000 \\
\hline 76 & gamma & 1 & 22 & 64 & 0 & 0 & -.01012 & .06728 & .03265 & .07547 & .00000 \\
\hline 77 & gamma & 1 & 22 & 64 & 0 & 0 & -.13812 & .07081 & -.07217 & .17117 & .00000 \\
\hline 78 & gamma & 1 & 22 & 66 & 0 & 0 & -.14093 & -.11124 & -.09549 & .20335 & .00000 \\
\hline 79 & gamma & 1 & 22 & 66 & 0 & 0 & -.03282 & -.12420 & .01347 & .12917 & .00000 \\
\hline 80 & gamma & 1 & 22 & 67 & 0 & 0 & -1.91607 & -.95766 & 2.68075 & 3.43145 & .00000 \\
\hline 81 & gamma & 1 & 22 & 67 & 0 & 0 & -2.06484 & -1.15251 & 3.10932 & 3.90637 & .00000 \\
\hline 82 & gamma & 1 & 22 & 68 & 0 & 0 & -.91460 & -1.29394 & 1.48769 & 2.17348 & .00000 \\
\hline 83 & (pio) & 11 & 111 & 68 & 84 & 85 & -1.53274 & -.79375 & 1.62187 & 2.37235 & .13500 \\
\hline 84 & gamma & 1 & 22 & 83 & 0 & 0 & -1.05165 & -.47797 & 1.05048 & 1.56138 & .00000 \\
\hline 85 & gamma & 1 & 22 & 83 & 0 & 0 & -.48109 & -.31578 & .57140 & .81096 & .00000 \\
\hline & & sum charge: & .00 & sum momentum & and & mass: & .00000 & .00000 & .00001 & 180.00000 & 180.00000 \\
\hline
\end{tabular}

\begin{tabular}{|c|c|c|c|c|c|}
\hline * & & Windor V & & & * \\
\hline * & & VESKO FINAL REPORT & & & * \\
\hline * & & mode 1 & & & * \\
\hline * & 18.41936258 & approx xs_crude VESK & XCVESK & V4 & * \\
\hline * & 18.22119473 & .01813815 exact $x s_{-}$crude VESK & XSVE & V5 & * \\
\hline * & 18.23931434 & .00000000 exact xs_crude Gaus & XSGS & V6 & * \\
\hline * & .00987144 & XCVESK_appr/XSGS-1 & & V7 & * \\
\hline * & $-.00099344+-$ & .00099644 XSVE_exact/XSGS-1 & & V8 & * \\
\hline
\end{tabular}

\begin{tabular}{|c|c|c|c|c|c|}
\hline * & & KARLUD FIIAL REPORT & & & * \\
\hline * & & Hindor A & & & * \\
\hline * & & & & & * \\
\hline * & $X$ sect & with NO Matrix Element & & & * \\
\hline * & 4913 & total no of events & NEVTOT & AO & * \\
\hline * & 0 & WTcrud $<0$ evts & HEVNEG & A 1 & * \\
\hline * & 18.95197787 & sigma_crude & XCRUDE & A 2 & * \\
\hline * & .85612580 & $.00507362\langle W T c r u d\rangle$ rel err & WTKACR & A3 & * \\
\hline * & 16. 22527727 & .08232088 sigma, no Matrix El. & XSKR & A4 & * \\
\hline * & & & & & * \\
\hline * & & & & & * \\
\hline * & Xsect rith Born & Matrix El. only, NO Betas & & & * \\
\hline * & 4913 & total no of events & NEVTOT & A5 & * \\
\hline * & 0 & WTcrud*WTborn $<0$ evt & NEVNEG & A 6 & * \\
\hline * & .78111712 & $.01158884\langle W T c r u d * W T$ born>, rel & WTKABO & A7 & * \\
\hline * & 14.80371432 & .17155790 sigma (Born M.El.) & XSKAO & A8 & * \\
\hline * & & & & & * \\
\hline * & & Xsect OVER Wtmax & & & * \\
\hline * & Born & Matrix El. only, no Betas & & & * \\
\hline * & 0 & no of ev OVER Htmax & NEVACC & A9 & * \\
\hline * & .00000000 & sigma OVER ntmax & XSKABO & A 10 & * \\
\hline * & .00000000 & relat sigma OVER & XSKABO & A 11 & * \\
\hline
\end{tabular}




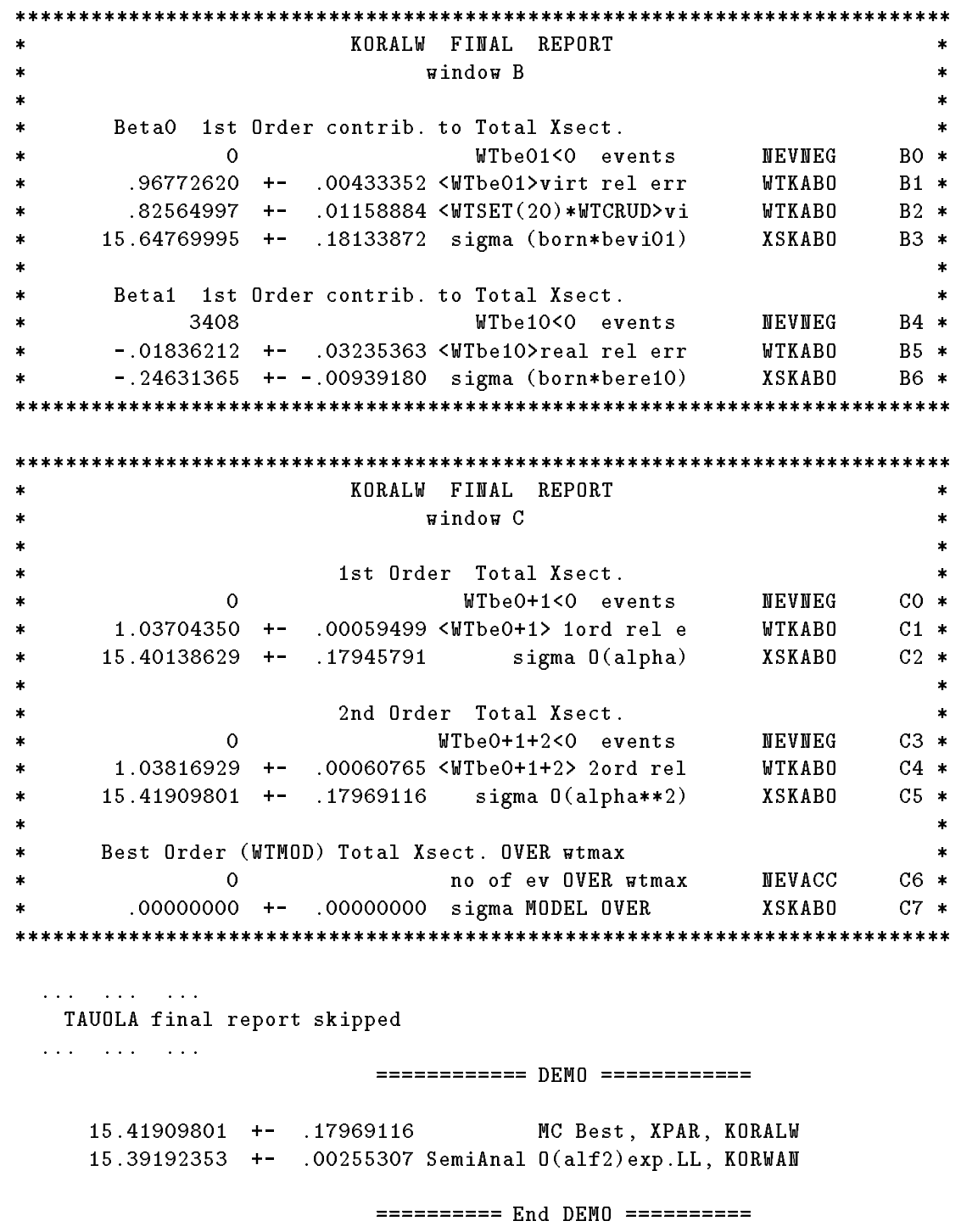

\section{References}

[1] M. Skrzypek et al., Initial-state QED corrections to W-pair production at LEP2/NLC - Monte Carlo versus semianalytical approach, CERN preprint CERN-TH/95-246 (unpublished).

[2] S. Jadach and B. F. L. Ward, Comput. Phys. Commun. 56, 351 (1990).

[3] S. Jadach, B. F. L. Ward, and Z. Wạs, Comput. Phys. Commun. 79, 503 (1994).

[4] D. R. Yennie, S. Frautschi, and H. Suura, Ann. Phys. (NY) 13, 379 (1961).

[5] S. Jadach and B. F. L. Ward, Phys. Lett. B274, 470 (1992). 
[6] J. Fleischer, F. Jegerlehner, K. Kołodziej, and G. J. van Oldenborgh, Comput. Phys. Commun. 85, 29 (1995).

[7] G. J. van Oldenborgh, preprint INLO-PUB-95/04, hep-ph/9503353 (unpublished).

[8] K. Hagiwara, R. Peccei, D. Zeppenfeld, and K. Hikasa, Nucl. Phys. B282, 253 (1987).

[9] K. Kołodziej and M. Zrałek, Phys. Rev. D43, 3619 (1991).

[10] S. Jadach, E. Richter-Wạs, B. F. L. Ward, and Z. Wạs, Comput. Phys. Commun. 70, 305 (1992).

[11] J. Jackson, Classical Electrodynamics (Wiley, New York, 1975).

[12] S. Jadach, E. Richter-Wạs, B. F. L. Ward, and Z. Wąs, Phys. Lett. B268, 253 (1991).

[13] S. Jadach, E. Richter-Wa̧s, B. F. L. Ward, and Z. Wạs, Phys. Lett. B353, 362 (1995), CERN preprint CERN-TH/95-38.

[14] M. Skrzypek, Acta Phys. Pol. B23, 135 (1992).

[15] T. Muta, R. Najima, and S. Wakaizumi, Mod. Phys. Lett. A1, 203 (1986).

[16] S. Jadach, M. Skrzypek, and B. Ward, Phys. Lett. B257, 173 (1991).

[17] E. Kuraev and V. Fadin, Sov. J. Nucl. Phys. 41, 466 (1985).

[18] S. Jadach, The FORTRAN code GLIBK, 1995 (unpublished).

[19] R. Decker, S. Jadach, J. Kühn, and Z. Wa̧s, Comput. Phys. Commun. 76, 361 (1993).

[20] E. Barberio and Z. Wa̧s, Comput. Phys. Commun. 79, 291 (1994).

[21] T. Sjöstrand and M. Bengtsson, Comput. Phys. Commun. 43, 367 (1987).

[22] M. Aguilar-Benitez et al., Phys. Rev. D50, 1173 (1994).

[23] S. Jadach and Z. Wa̧s, Comput. Phys. Commun. 85, 453 (1995).

[24] J. D. Bjorken and S. D. Drell, Relativistic Quantum Mechanics (McGraw-Hill, New York, 1964).

[25] J. Fleischer, F. Jegerlehner, and M. Zrałek, Z. Phys. C42, 409 (1989). 